\title{
RESIDUES AND FILTERED D-MODULES
}

\author{
CHRISTIAN SCHNELL
}

\begin{abstract}
For an embedding of sufficiently high degree of a smooth projective variety $X$ into projective space, we use residues to define a filtered holonomic $\mathcal{D}$-module $(\mathcal{M}, F)$ on the dual projective space. This gives a concrete description of the intermediate extension to a Hodge module on $P$ of the variation of Hodge structure on the middle-dimensional cohomology of the hyperplane sections of $X$. We also establish many results about the sheaves $F_{k} \mathcal{M}$, such as positivity, vanishing theorems, or reflexivity.
\end{abstract}

\section{A. Overview}

1. Introduction. Let $X$ be a complex projective manifold of dimension $n$, and let $D \subseteq X$ be a smooth and very ample hypersurface. The cohomology of the complement $X \backslash D$ can be represented by meromorphic forms on $X$ with poles along $D$. It also carries a mixed Hodge structure, and Griffiths [Gri69] (for $X=\mathbb{P}^{n}$ ) and Green Gre85. (in general) have shown that the Hodge filtration is basically the filtration by pole order, provided that the line bundle $\mathscr{O}_{X}(D)$ is sufficiently ample. One consequence is a formula for the Hodge filtration on the vanishing cohomology $H_{e v}^{n-1}(D, \mathbb{Q})$ of the hypersurface (see $\$ 2$ below).

The purpose of this article is to generalize the above picture to the case when $D$ is allowed to have singularities. Fix a very ample line bundle $\mathscr{O}_{X}(1)$ on $X$, and consider all hypersurfaces, smooth or singular, that belong to the linear system $P=\left|\mathscr{O}_{X}(1)\right|$. Using residues, we construct a $\mathcal{D}$-module $\mathcal{M}$ on the projective space $P$, together with a good filtration $F=F \cdot \mathcal{M}$ by $\mathscr{O}_{P}$-coherent subsheaves. Provided that $\mathscr{O}_{X}(1)$ is sufficiently ample, we then show that $(\mathcal{M}, F)$ is regular and holonomic, and in fact underlies a polarized Hodge module on $P$. We also show that the coherent sheaves $F_{k} \mathcal{M}$ have many remarkable properties. The $\mathcal{D}$-module $\mathcal{M}$ is intimately related to the geometry of the incidence variety $\mathscr{X} \subseteq P \times X$, and in the process of describing $\mathcal{M}$, we recover the results about $\mathscr{X}$ obtained by Brosnan, Fang, Nie, and Pearlstein in BFNP07. We also strengthen one of their theorems, by showing that, in the Decomposition Theorem, no terms with support in the dual variety appear once the vanishing cohomology of the hypersurfaces is nontrivial.

These results seem interesting for two reasons. One is theoretical: Computing minimal extensions of holonomic $\mathcal{D}$-modules is a difficult problem, except in the case of a divisor with normal crossing singularities. In the situation above, we have a flat vector bundle with fibers $H_{e v}^{n-1}(D, \mathbb{C})$ on the complement of the discriminant locus $P \backslash X^{\vee}$, underlying the evident variation of Hodge structure. Our $\mathcal{D}$-module $\mathcal{M}$ is in fact the minimal extension of that flat vector bundle, and so we have a very

2000 Mathematics Subject Classification. 14D07; 32C38, 14F10.

Key words and phrases. Residues, Hypersurfaces, Filtered D-module, Mixed Hodge module. 
explicit description of the minimal extension via residues, despite the fact that the divisor $X^{\vee}$ is typically very singular.

The other reason is practical: The Hodge filtration on a minimal extension can be used very nicely in the construction of certain analytic spaces, the main example being the construction of complex-analytic Néron models for families of intermediate Jacobians Sch09b. Good properties of the sheaves in the filtration (such as positivity or reflexivity) translate directly into good properties of the resulting analytic spaces (such as holomorphic convexity or control over singularities). In a forthcoming paper, we will use the results about the sheaves $F_{k} \mathcal{M}$ obtained here to give a precise description of the Néron model for the family of intermediate Jacobians $J^{n-1}(D)$, for $n=\operatorname{dim} X$ even. Other applications are to the study of Noether-Lefschetz loci in $P$ (for $n$ odd).

2. Summary of results. Before outlining the results of this paper, we shall briefly review the theorem of Griffiths and Green. Keeping the notation of \$1 the Lefschetz theorems show that the cohomology groups of $D$ can be obtained from those of $X$ with the exception of $H^{n-1}(D, \mathbb{Q})$; the latter is the direct sum of $H^{n-1}(X, \mathbb{Q})$ and the so-called vanishing cohomology

$$
H_{e v}^{n-1}(D, \mathbb{Q})=\operatorname{ker}\left(i_{*}: H^{n-1}(D, \mathbb{Q}) \rightarrow H^{n+1}(X, \mathbb{Q})\right),
$$

the kernel of the Gysin map for the inclusion $i: D \hookrightarrow X$. The vanishing cohomology of $D$ is related to the cohomology of the open complement $X \backslash D$ through the exact sequence

$$
0 \longrightarrow H_{0}^{n}(X, \mathbb{Q}) \longrightarrow H^{n}(X \backslash D, \mathbb{Q}) \longrightarrow H_{e v}^{n-1}(D, \mathbb{Q}) \longrightarrow 0
$$

in which $H_{0}^{n}(X, \mathbb{Q})=\operatorname{ker}\left(i^{*} \circ i_{*}: H^{n}(X, \mathbb{Q}) \rightarrow H^{n+2}(X, \mathbb{Q})\right)$ denotes the primitive cohomology of $X$. The map $H^{n}(X \backslash D, \mathbb{Q}) \rightarrow H_{e v}^{n-1}(D, \mathbb{Q})$ is the well-known residue map, whose analytic description is as follows: by a theorem of Grothendieck's, the cohomology groups of $X \backslash D$ are computed by holomorphic forms on $X \backslash D$ with at worst poles along the divisor $D$, and the residue map takes such a form to its residue along $D$.

All three groups in (2.1) carry mixed Hodge structures, and through the work of Griffiths Gri69 (for the case $X=\mathbb{P}^{n}$ ) and Green Gre85, it is known how to compute their Hodge filtrations: they are essentially given by pole order. That is to say, if we let $\Omega_{X}^{p}(* D)$ denote the sheaf of meromorphic $p$-forms on $X$ with poles along $D$, and $\Omega_{X}^{p}(k D)$ the subsheaf of those with a pole of order at most $k$, then

$$
H^{n}(X \backslash D, \mathbb{C}) \simeq \frac{H^{0}\left(X, \Omega_{X}^{n}(* D)\right)}{d H^{0}\left(X, \Omega_{X}^{n-1}(* D)\right)},
$$

and the Hodge filtration is (for $k \geq 1$ ) given by

$$
F^{n+1-k} H^{n}(X \backslash D, \mathbb{C}) \simeq \frac{H^{0}\left(X, \Omega_{X}^{n}(k D)\right)}{d H^{0}\left(X, \Omega_{X}^{n-1}((k-1) D)\right)} .
$$

In both formulas, $d$ stands for the exterior derivative on forms. The second one holds as long as the line bundle $\mathscr{O}_{X}(D)$ is sufficiently ample; more precisely, one needs that $H^{q}\left(X, \Omega_{X}^{p}(k)\right)=0$ for $p \geq 0$ and $q, k>0$. From (2.1), one obtains the following formula for the Hodge filtration on the vanishing cohomology of $D$ :

$$
F^{n-k} H_{e v}^{n-1}(D, \mathbb{C}) \simeq \frac{H^{0}\left(X, \Omega_{X}^{n}(k D)\right)}{F^{n+1-k} H_{0}^{n}(X, \mathbb{C})+d H^{0}\left(X, \Omega_{X}^{n-1}((k-1) D)\right)} .
$$


The isomorphism in (2.2) is compatible with moving $D$ in the linear system $P$, and provides a very convenient description for the resulting variation of Hodge structure. Consider the incidence variety $\mathscr{X} \subseteq P \times X$, which is a projective bundle over $X$ and hence nonsingular. From now on, we denote the individual hypersurfaces by $\mathscr{X}_{p}=\pi^{-1}(p)$, where $\pi: \mathscr{X} \rightarrow P$ is the projection to the first factor. Let $\pi^{s m}: \mathscr{X}^{s m} \rightarrow P^{s m}$ be the restriction of $\pi$ to the open subset $P^{s m}$ over which $\pi$ is smooth, and let $j: P^{s m} \hookrightarrow P$ denote the inclusion map.

On $P^{s m}$, we have the variation of Hodge structure

$$
\mathscr{H}=R_{e v}^{n-1} \pi_{*}^{s m} \mathbb{Q}=\operatorname{ker}\left(R^{n-1} \pi_{*}^{s m} \mathbb{Q} \rightarrow H^{n+1}(X, \mathbb{Q})\right),
$$

whose fibers are the weight $n-1$ rational Hodge structures on $H_{e v}^{n-1}\left(\mathscr{X}_{p}, \mathbb{Q}\right)$. Let $\mathscr{H}_{\mathscr{O}}$ be the underlying holomorphic vector bundle, and let $F^{k} \mathscr{H}_{\mathscr{O}}$ be the holomorphic subbundles $F^{k} \mathscr{H}_{\mathscr{O}}$ given by the Hodge filtration. The Gauss-Manin connection $\nabla$ makes $\mathscr{H}_{\mathscr{O}}$ into a flat vector bundle, and the Hodge bundles satisfy Griffiths' transversality condition $\nabla\left(F^{k} \mathscr{H}_{\mathscr{O}}\right) \subseteq \Omega_{P s m}^{1} \otimes F^{k-1} \mathscr{H}_{\mathscr{O}}$. Now the isomorphism in (2.2) means that $F^{n-k} \mathscr{H}_{\mathscr{O}}$ is a quotient of the vector bundle

$$
\pi_{*}^{s m} \Omega_{P^{s m} \times X / P^{s m}}^{n}\left(k \mathscr{X}^{s m}\right) \simeq H^{0}\left(X, \Omega_{X}^{n}(k)\right) \otimes \mathscr{O}_{P^{s m}}(k),
$$

in a way that is compatible with differentation.

Trying to extend this description to singular hypersurfaces naturally leads to filtered $\mathcal{D}$-modules. To avoid the problems caused by the singularities, we use the fact that the incidence variety $\mathscr{X}$ is nonsingular, and define coherent subsheaves $F_{k} \mathcal{M}$ of the quasi-coherent sheaf $j_{*} \mathscr{H}_{\mathscr{O}}$ by the following rule: for any open set $U \subseteq P$, a section $s \in \Gamma\left(U \cap P^{s m}, \mathscr{H}_{\mathscr{O}}\right)$ shall belong to $\Gamma\left(U, F_{k} \mathcal{M}\right)$ if and only if there exists a meromorphic $n$-form $\omega \in \Gamma\left(U \times X, \Omega_{P \times X}^{n}(k \mathscr{X})\right)$ such that

$$
s(p)=\operatorname{Res} \mathscr{X}_{p}\left(\left.\omega\right|_{X \backslash \mathscr{X}_{p}}\right)
$$

for any $p \in U \cap P^{s m}$. The result of Griffiths and Green shows that, once $\mathscr{O}_{X}(1)$ is sufficiently ample, the coherent sheaf $F_{k} \mathcal{M}$ is a natural extension of the vector bundle $F^{n-k} \mathscr{H}_{\mathscr{O}}$ from $P^{s m}$ to $P$. Now let $\mathcal{M}$ be the union of the $F_{k} \mathcal{M}$ inside $j_{*} \mathscr{H}_{\mathscr{O}}$. It is then not hard to show that $(\mathcal{M}, F)$ is a coherent filtered $\mathcal{D}_{P}$-module, whose restriction to $P^{s m}$ is the flat vector bundle $\left(\mathscr{H}_{\mathscr{O}}, \nabla\right)$ with its Hodge filtration (see (8). The following theorem summarizes our main results.

Theorem A. If the vanishing cohomology of the hypersurfaces is nontrivial, meaning that $\mathscr{H}_{\mathscr{O}} \neq 0$, then the filtered $\mathcal{D}$-module $(\mathcal{M}, F)$ has the following properties:

(1) $\mathcal{M}$ is regular and holonomic, and is the minimal extension of the flat vector bundle $\left(\mathscr{H}_{\mathscr{O}}, \nabla\right)$ from $P^{s m}$ to $P$.

(2) $\left(\mathcal{M}, F_{\bullet+n}\right)$ underlies the polarized Hodge module obtained by intermediate extension of the variation of Hodge structure $R_{e v}^{n-1} \pi_{*}^{s m} \mathbb{Q}$, provided that $H^{q}\left(X, \Omega_{X}^{p}(k)\right)=0$ for every $p \geq 0$ and every $q, k>0$.

(3) By the Riemann-Hilbert correspondence, the de Rham complex $\operatorname{DR}_{P}(\mathcal{M})$ is constructible, and its cohomology sheaves satisfy

$$
R^{n-1+k} \pi_{*} \mathbb{C} \simeq \mathcal{H}^{k-d} \operatorname{DR}_{P}(\mathcal{M}) \oplus H^{n-1-k}(X, \mathbb{C})
$$

for all $0 \leq k \leq d$.

Note. The conditions in the theorem are always satisfied if $\mathscr{O}_{X}(1)$ is a sufficiently high power of a very ample line bundle. 
In the process of proving Theorem A we compute the characteristic variety of the $\mathcal{D}$-module $\mathcal{M}$ inside the cotangent bundle of $P$. Before we state the result, recall that $\operatorname{Gr}^{F} \mathcal{D}_{P} \simeq$ Sym $\Theta_{P}$ is the symmetric algebra on the tangent bundle of $P$; consequently, the graded module $\mathrm{Gr}^{F} \mathcal{M}$ defines a coherent sheaf on $T_{P}^{*}$, whose support is by definition the characteristic variety. Because of the grading, we also get a coherent sheaf on the projectivized cotangent bundle $\mathbb{P}\left(\Theta_{P}\right)$. One can show (see 6 ) that the incidence variety $\mathscr{X}$ embeds into $\mathbb{P}\left(\Theta_{P}\right)$. Denote by $\mathscr{Y} \subseteq \mathscr{X}$ the union of all the singular points in the hypersurfaces $\mathscr{X}_{p}$; then the second projection $\psi: \mathscr{Y} \rightarrow X$ is again a projective bundle, and so $\mathscr{Y}$ is also a smooth subvariety of $\mathbb{P}\left(\Theta_{P}\right)$. The following theorem relates it to the characteristic variety of $(\mathcal{M}, F)$.

Theorem B. If the vanishing cohomology of the hypersurfaces is nontrivial, then the coherent sheaf on $\mathbb{P}\left(\Theta_{P}\right)$ defined by the graded Sym $\Theta_{P}$-module $\operatorname{Gr}^{F} \mathcal{M}$ is precisely $\psi^{*} \omega_{X}$.

We note that this result fits into the context of Fourier-Mukai transforms for filtered $\mathcal{D}$-modules Lau85, in this case between $X$ and on $P$. That is to say, if we transform $\mathscr{O}_{X}$ (with the trivial filtration $\operatorname{Gr}_{0}^{F} \mathscr{O}_{X}=\mathscr{O}_{X}$ ) by the kernel $\mathscr{O}_{P \times X}(* \mathscr{X})$, then $(\mathcal{M}, F)$ is a direct summand in one of the cohomology modules of the resulting complex. More generally, because of the embedding $i: X \hookrightarrow Q$, this may be seen as a special case of the Fourier-Mukai transform between filtered $\mathcal{D}$-modules on the projective space $Q$ and its dual $P$. (It appears that this observation is originally due to Beilinson.)

This computation also leads to the following result about the terms that appear in the Decomposition Theorem for the direct image of $\mathbb{Q}\left[d_{\mathscr{X}}\right]$ under the morphism $\pi: \mathscr{X} \rightarrow P$.

Theorem C. If the vanishing cohomology of the hypersurfaces is nontrivial, then each piece in the decomposition of the polarized Hodge modules $H^{k} \pi_{*} \mathbb{Q}_{\mathscr{X}}^{H}\left[d_{\mathscr{X}}\right]$ has strict support equal to all of $P$.

As explained above, we view the coherent sheaves $F_{k} \mathcal{M}$ (especially in the range $1 \leq k \leq n$ ) as being natural extensions of the Hodge bundles $F^{n-k} \mathscr{H}_{\mathscr{O}}$. The concrete description by residues allows us to show that they have many nice properties.

Theorem D. Suppose that we have $H^{q}\left(X, \Omega_{X}^{p}(k)\right)=0$ for $k, q>0$ and $p \geq 0$. Then the coherent sheaves $F_{k} \mathcal{M}$ have the following properties:

(1) $F_{1} \mathcal{M}$ is an ample vector bundle, and is a direct summand of $\pi_{*} \mathscr{O}_{\mathscr{X}}\left(K_{\mathscr{X} / P}\right)$.

(2) $H^{i}\left(P, \Omega_{P}^{p} \otimes F_{k} \mathcal{M}\right)=0$ for every $i \geq \max (p, 0)$.

(3) For $0 \leq i \leq \operatorname{dim} P-1$, we have

$$
\operatorname{Ext}_{p}^{i}\left(F_{k} \mathcal{M}, \mathscr{O}_{P}\right) \simeq\left(\frac{F^{n+1-k} H^{n+1-i}(X, \mathbb{C})}{F^{n-k} H^{n-1-i}(X, \mathbb{C})}\right)^{\vee}
$$

(4) Given $m \geq 1$, the sheaves $\operatorname{Gr}_{k}^{F} \mathcal{M}$ and $F_{k} \mathcal{M}$ in the range $1 \leq k \leq n$ satisfy a variant of Serre's condition $S_{m}$, provided that $\mathscr{O}_{X}(1)$ is a sufficiently high power of a very ample line bundle. In particular, they are reflexive.

The proof of (4) given in $₫ 22$ depends on two things: a general duality result for filtered Cohen-Macaulay $\mathcal{D}$-modules [Sch09a, and the fact that the set of hypersurfaces with "many" singularities has very high codimension in $P$ if $\mathscr{O}_{X}(1)$ is a sufficiently large power of a very ample line bundle. 
3. Acknowledgements. This work is part of my Ph.D. thesis, and it is my pleasure to thank my former adviser Herb Clemens for his help and his kindness during my time in graduate school. Needless to say, the idea to study the behavior of the residue map near singular hypersurfaces is due to him.

I also acknowledge the influence of Patrick Brosnan and Greg Pearlstein, whose paper [BFNP07] with Hao Fang and Zhaohu Nie appeared at about the time when I was thinking about residues, and led me to describe the $\mathcal{D}$-module $(\mathcal{M}, F)$ using the theory of mixed Hodge modules.

I am grateful to Joel Kamnitzer and Sabin Cautis for useful conversations about Fourier-Mukai transforms for filtered $\mathcal{D}$-modules, and for calling my attention to Laumon's work Lau85.

Lastly, my thanks go to Paul Taylor for providing the package diagrams that was used to typeset the many commutative diagrams in the paper.

4. Conventions. In dealing with filtrations, we index increasing filtrations (such as weight filtrations, or Hodge filtrations on $\mathcal{D}$-modules) by lower indices, and decreasing filtrations (such as Hodge filtrations on vector spaces, or $V$-filtrations on left $\mathcal{D}$-modules) by upper indices. We may pass from one to the other by the convention that $F^{\bullet}=F_{-} \bullet$. To be consistent, shifts in the filtration thus have different effects in the two cases:

$$
F[1]^{\bullet}=F^{\bullet+1}, \quad \text { while } \quad F[1] \bullet=F_{\bullet-1} .
$$

This convention agrees with the notation used in M. Saito's papers.

In this paper, we work with left $\mathcal{D}$-modules, and $\mathcal{D}$-module always means left $\mathcal{D}$-module (in contrast to Sai90, where right $\mathcal{D}$-modules are used).

When $M$ is a mixed Hodge module, the effect of a Tate twist $M(k)$ on the underlying filtered $\mathcal{D}$-module $(\mathcal{M}, F)$ is as follows:

$$
(\mathcal{M}, F)(k)=(\mathcal{M}, F[k])=\left(\mathcal{M}, F_{\bullet-k}\right) .
$$

For a regular holonomic $\mathcal{D}$-module $\mathcal{M}$ that is defined on the complement of a divisor $D \subseteq X$, we let $j_{*}^{\text {reg }} \mathcal{M}$ be the direct image in the category of regular holonomic $\mathcal{D}$-modules; its sections have poles of finite order along $D$.

The individual sections of the paper are numbered consecutively, and are referred to with a paragraph symbol (such as $\$ 4$ ).

\section{B. Residues AND FILTERED D-MOdules}

Throughout, we let $X$ be a smooth projective variety of dimension $n$, and $\mathscr{O}_{X}(1)$ a very ample line bundle, embedding $X$ into the projective space $Q=\mathbb{P}(V)$, where $V=H^{0}\left(X, \mathscr{O}_{X}(1)\right)$. We denote by $P$ the dual projective space, parametrizing hyperplane sections of $X$ for the given embedding, and let $\pi: \mathscr{X} \rightarrow P$ be the universal hypersurface.

5. Filtered $\mathcal{D}$-modules on projective space. Let $V$ be a complex vector space of dimension $d+1$, and set $P=\mathbb{P}\left(V^{\vee}\right)$, which is a projective space of dimension $d$. Let $\mathcal{D}_{P}$ denote the sheaf of differential operators on $P$; it is naturally filtered, with the subsheaf $F_{m} \mathcal{D}_{P}$ consisting of those differential operators whose order is at most $m$. The associated graded sheaf

$$
\operatorname{Gr}^{F} \mathcal{D}_{P}=\bigoplus_{m=0}^{\infty} F_{m} \mathcal{D}_{P} / F_{m-1} \mathcal{D}_{P}
$$


is commutative, and in fact isomorphic to the symmetric algebra on the tangent bundle Sym $\Theta_{P}$. Recall that a left $\mathcal{D}$-module on $P$ is a quasi-coherent sheaf $\mathcal{M}$ with a left action by $\mathcal{D}_{P}$. Moreover, a filtered $\mathcal{D}$-module is a pair $(\mathcal{M}, F)$, consisting of left a $\mathcal{D}$-module $\mathcal{M}$ together with an increasing filtration $F=F_{\bullet} \mathcal{M}$ by $\mathscr{O}_{P}$-coherent subsheaves that is bounded from below, and satisfies

$$
F_{m} \mathcal{D}_{P} \cdot F_{k} \mathcal{M} \subseteq F_{m+k} \mathcal{M}
$$

for all $k, m \in \mathbb{Z}$. One says that the filtration $F$ is good if the inclusion in (5.1) is an equality for large values of $k$; this is equivalent to

$$
\operatorname{Gr}^{F} \mathcal{M}=\bigoplus_{k \in \mathbb{Z}} F_{k} \mathcal{M} / F_{k-1} \mathcal{M}
$$

being finitely generated over $\operatorname{Sym} \Theta_{P}$ by $\left[\mathrm{BGK}^{+} 87\right.$, Theorem 4.4]. Note that $\mathcal{M}$ is coherent as an $\mathscr{O}_{P}$-module if, and only if, it is locally free of finite rank (and hence a flat vector bundle); see [BGK ${ }^{+} 87$, Proposition 1.7].

Sym $\Theta_{P}$ is naturally the sheaf of functions on the cotangent bundle of $P$, and so any filtered coherent $\mathcal{D}$-module $(\mathcal{M}, F)$ defines a coherent sheaf on $T_{P}^{*}$; its support is by definition the characteristic variety $S S(\mathcal{M}, F)$. Again, the characteristic variety is contained in the zero section exactly when $\mathcal{M}$ is locally free of finite rank.

Because of the grading, $S S(\mathcal{M}, F)$ is a cone in $T_{P}^{*}$, and each component of this cone has dimension at least $d=\operatorname{dim} P$, a fact known as Bernstein's inequality $\mathrm{BGK}^{+} 87$, Theorem 1.10]. The filtered $\mathcal{D}$-module $(\mathcal{M}, F)$ is said to be holonomic if its characteristic variety is of pure dimension $d$. For every holonomic $\mathcal{D}$-module $\mathcal{M}$, there is a finite union of closed subvarieties of $P$, outside of which $\mathcal{M}$ is a flat vector bundle, namely the image of all those components of $S S(\mathcal{M}, F)$ that are not contained in the zero section. For a holonomic $\mathcal{D}$-module $\mathcal{M}$, the multiplicity of the sheaf $\operatorname{Gr}^{F} \mathcal{M}$ along each component of the characteristic variety is the same for every good filtration $F$. These multiplicities are additive in short exact sequences of holonomic $\mathcal{D}$-modules, which implies that the category of holonomic $\mathcal{D}$-modules is Artinian: every holonomic module admits a finite filtration with simple holonomic subquotients. This leads to the following notion: given a holonomic $\mathcal{D}$-module $\mathcal{N}$ on $P \backslash Z$, we say that a holonomic $\mathcal{D}$-module $\mathcal{M}$ is the minimal extension of $\mathcal{N}$ if $\left.\mathcal{M}\right|_{P \backslash Z} \simeq \mathcal{N}$, and if $\mathcal{M}$ has no nontrivial submodules or quotient modules whose support is contained in $Z$.

Given a filtered coherent $\mathcal{D}$-module $(\mathcal{M}, F)$, the graded Sym $\Theta_{P}$-module $\operatorname{Gr}^{F} \mathcal{M}$ naturally defines a coherent sheaf on the projectivized cotangent bundle $\mathbb{P}\left(\Theta_{P}\right)=$ $\operatorname{Proj}\left(\operatorname{Sym} \Theta_{P}\right)$; we shall call it the characteristic sheaf of $(\mathcal{M}, F)$, and denote it by $\mathscr{C}(\mathcal{M}, F)$. We recover the characteristic variety by taking the cone over the support of the characteristic sheaf, together with possibly the zero section; in particular, one can compute the multiplicity along the components of the characteristic variety from $\mathscr{C}(\mathcal{M}, F)$.

A second interpretation will be useful in what follows. Let $Q=\mathbb{P}(V)$, and note that the two projective spaces $P$ and $Q$ are dual to each other, with points $p \in P$ corresponding to hyperplanes $H_{p} \subseteq Q$. The incidence variety

$$
\mathscr{Q}=\left\{(p, x) \in P \times Q \mid x \in H_{p}\right\}
$$

is a nonsingular hypersurface, with line bundle $\mathscr{O}_{P \times Q}(\mathscr{Q}) \simeq p r_{P}^{*} \mathscr{O}_{P}(1) \otimes p r_{Q}^{*} \mathscr{O}_{Q}(1)$. It is naturally isomorphic to the projectivized cotangent bundle $\mathbb{P}\left(\Theta_{P}\right)$; indeed, the 
exactness of the Euler sequence

$$
0 \longrightarrow \mathscr{O}_{P} \longrightarrow \mathscr{O}_{P}(1) \otimes V \longrightarrow \Theta_{P} \longrightarrow 0
$$

on the projective space $P$ implies that $\mathbb{P}\left(\Theta_{P}\right)$ embeds into $\mathbb{P}\left(\mathscr{O}_{P}(1) \otimes V\right)=P \times Q$, with line bundle $p r_{P}^{*} \mathscr{O}_{P}(1) \otimes p r_{Q}^{*} \mathscr{O}_{Q}(1)$, and a moment's thought shows that the image is precisely $\mathscr{Q}$.

Let $i: \mathscr{Q} \hookrightarrow P \times Q$ be the inclusion. The line bundle $\mathscr{O}_{P \times Q}(\mathscr{Q})$ is very ample, and embeds $P \times Q$ into a larger projective space; we let

$$
S=\bigoplus_{k=0}^{\infty} H^{0}\left(\mathscr{Q}, i^{*} \mathscr{O}_{P \times Q}(k \mathscr{Q})\right)
$$

be the homogeneous coordinate ring in this embedding, so that $\mathbb{P}\left(\Theta_{P}\right) \simeq$ Proj $S$. Not surprisingly, we have an isomorphism of graded rings

$$
S \simeq H^{0}\left(P, \operatorname{Sym} \Theta_{P}\right)
$$

in fact, after pushing forward the exact sequence

$$
0 \longrightarrow \mathscr{O}_{P \times Q}((k-1) \mathscr{Q}) \longrightarrow \mathscr{O}_{P \times Q}(k \mathscr{Q}) \longrightarrow i^{*} \mathscr{O}_{P \times Q}(k \mathscr{Q}) \longrightarrow 0
$$

to $P$, and using the information coming from the Euler sequence, we find that

$$
H^{0}\left(\mathscr{Q}, i^{*} \mathscr{O}_{P \times Q}(k \mathscr{Q})\right) \simeq H^{0}\left(P, \frac{\mathscr{O}_{P}(k) \otimes \operatorname{Sym}^{k} V}{\mathscr{O}_{P}(k-1) \otimes \operatorname{Sym}^{k-1} V}\right) \simeq H^{0}\left(P, \operatorname{Sym}^{k} \Theta_{P}\right)
$$

for all $k \geq 0$. It follows from these observations that the characteristic sheaf $S S(\mathcal{M}, F)$ of a filtered coherent $\mathcal{D}$-module $(\mathcal{M}, F)$ is the coherent sheaf on Proj $S$ associated to the graded $S$-module

$$
C(\mathcal{M}, F)=\bigoplus_{k \in \mathbb{Z}} H^{0}\left(P, F_{k} \mathcal{M} / F_{k-1} \mathcal{M}\right)=H^{0}\left(P, \operatorname{Gr}^{F} \mathcal{M}\right)
$$

We shall refer to it as the characteristic module of $(\mathcal{M}, F)$; as usual, the characteristic sheaf is completely determined by the homogeneous components of $C(\mathcal{M}, F)$ in degrees $k \gg 0$.

6. The universal hyperplane section. Now let $X$ be a smooth projective variety of dimension $n$, and fix a very ample line bundle $\mathscr{O}_{X}(1)$ on $X$. We let $V=$ $H^{0}\left(X, \mathscr{O}_{X}(1)\right)$ be the space of its global sections, and therefore get a nondegenerate embedding $i: X \hookrightarrow Q$ into the projective space $Q=\mathbb{P}(V)$. The universal hyperplane section is the incidence variety

$$
\mathscr{X}=\left\{(p, x) \in P \times X \mid x \in H_{p} \cap X\right\} .
$$

We obviously have $\mathscr{X}=\mathscr{Q} \cap(P \times X)$ inside $P \times Q$, in the notation of $\$ 5$, Because $\mathscr{Q} \simeq \mathbb{P}\left(\Theta_{Q}\right)$, it follows that

$$
\mathscr{X} \simeq \mathbb{P}\left(i^{*} \Theta_{Q}\right)
$$

is itself a projective bundle of rank $d-1$ over $X$; consequently, $\mathscr{X}$ is a nonsingular very ample hypersurface in $P \times X$ of dimension $n+d-1$, with corresponding line bundle $\mathscr{O}_{P \times X}(\mathscr{X}) \simeq p r_{P}^{*} \mathscr{O}_{P}(1) \otimes p r_{X}^{*} \mathscr{O}_{X}(1)$. Let $\pi: \mathscr{X} \rightarrow P$ be the projection to the first coordinate; its fibers $\mathscr{X}_{p}=\pi^{-1}(p)$ are the hyperplane sections of $X$.

We shall also use the subvariety $\mathscr{Y} \subseteq \mathscr{X}$, defined by the condition

$$
\mathscr{Y}=\left\{(p, x) \in P \times X \mid H_{p} \text { is tangent to } X \text { at } x\right\} ;
$$


its points are exactly the singular points in the fibers of $\pi$. Note that $\mathscr{Y}$ is itself nonsingular of dimension $d-1$, since it is isomorphic to the projective bundle $\mathbb{P}\left(N_{X \mid Q}\right)$ over $X$; this follows from the exact sequence

$$
0 \longrightarrow \Theta_{X} \longrightarrow i^{*} \Theta_{Q} \longrightarrow N_{X \mid Q} \longrightarrow 0 .
$$

The dual variety $X^{\vee} \subseteq P$ is by definition the image $\pi(\mathscr{Y})$. If there are points in $P$ corresponding to hyperplane sections with just a single ordinary double point singularity, then $\mathscr{Y}$ is birational to $X^{\vee}$ and hence a resolution of singularities.

Note. Both $\mathscr{X}$ and $\mathscr{Y}$ can be considered as subvarieties of the projectivized cotangent bundle $\mathbb{P}\left(\Theta_{P}\right) \simeq \mathscr{Q}$. In what follows, we shall see that both varieties are the support of the characteristic sheaves of two natural filtered $\mathcal{D}$-modules on $P$.

7. An auxilliary $\mathcal{D}$-module. Having completed our discussion of the universal hyperplane section, we now turn to the problem of extending the residue calculus to singular hyperplane sections of $X$. As above, let $\pi: \mathscr{X} \rightarrow P$ be the universal hyperplane section. To begin with, we define

$$
\mathcal{N}=p r_{P *} \Omega_{P \times X / P}^{n}(* \mathscr{X}),
$$

which is a quasi-coherent sheaf on $P$ whose sections over an open set $U$ are relative rational $n$-forms on $U \times X$ with poles along $\mathscr{X}$. It has a natural increasing filtration by coherent subsheaves $F_{k} \mathcal{N}$, corresponding to the order of the pole; set

$$
F_{k} \mathcal{N}=p r_{P *} \Omega_{P \times X / P}^{n}(k \mathscr{X}) \simeq H^{0}\left(X, \Omega_{X}^{n}(k)\right) \otimes \mathscr{O}_{P}(k),
$$

as well as $F_{k} \mathcal{N}=0$ for $k \leq 0$ to avoid degenerate cases.

We observe that $\mathcal{N}$ is naturally a left $\mathcal{D}$-module, since the sheaf of differential operators $\mathcal{D}_{P}$ acts on $\mathcal{N}$ by differentiating the coefficients. More precisely, for $\omega \in \Gamma(U, \mathcal{N})$ a relative rational $n$-form on $U \times X$, and $\xi \in \Gamma\left(U, \Theta_{P}\right)$ a vector field, we define

$$
\left.\xi \cdot \omega=\xi^{\prime}\right\lrcorner d \omega \in \Gamma(U, \mathcal{N}),
$$

where $\xi^{\prime}$ is the natural horizontal lifting of $\xi$ to a vector field on $U \times X$, and $\left.\xi^{\prime}\right\lrcorner d \omega$ indicates contraction. It is not hard to prove the following: (1) for a holomorphic function $f$ on $U$, we have $(f \xi) \cdot \omega=f(\xi \cdot \omega)$, as well as $\xi \cdot(f \omega)=(\xi f) \cdot \omega+f(\xi \cdot \omega)$; (2) for a second vector field $\eta$, we have $[\xi, \eta] \cdot \omega=\xi \cdot(\eta \cdot \omega)-\eta \cdot(\xi \cdot \omega)$; (3) if $\omega$ is a section of $F_{k} \mathcal{N}$, then $\xi \cdot \omega$ is a section of $F_{k+1} \mathcal{N}$. The action by vector fields thus extends uniquely to an action by $\mathcal{D}_{P}$, and so $\mathcal{N}$ is a left $\mathcal{D}$-module; moreover, we have $F_{m} \mathcal{D}_{P} \cdot F_{k} \mathcal{N} \subseteq F_{m+k} \mathcal{N}$, where $F_{\bullet} \mathcal{D}_{P}$ is the order filtration on $\mathcal{D}_{P}$.

Lemma 7.1. $F_{\bullet} \mathcal{N}$ is a good filtration, and $(\mathcal{N}, F)$ is a coherent filtered $\mathcal{D}$-module.

Proof. To prove that the filtration is good, we need to show that $F_{1} \mathcal{D}_{P} \cdot F_{k} \mathcal{N}=$ $F_{k+1} \mathcal{N}$ for all sufficiently large $k$. This is equivalent to the surjectivity of the map

$$
\Theta_{P} \otimes F_{k} \mathcal{N} \rightarrow F_{k+1} \mathcal{N} / F_{k} \mathcal{N}
$$

a question which is local on $P$. Fix an arbitrary point $p \in P$, and let $s_{0}, s_{1}, \ldots, s_{d}$ be a basis for the vector space $H^{0}\left(X, \mathscr{O}_{X}(1)\right)$, with $\left[s_{0}\right]$ equal to the point $p$. Any local section of $F_{k} \mathcal{N}$ can now be written in the form

$$
\omega=\frac{\omega(t)}{\left(s_{0}+\sum t_{i} s_{i}\right)^{k}},
$$


for some holomorphic map $\omega(t)$ from $\mathbb{C}^{d}$ into $H^{0}\left(X, \Omega_{X}^{n}(k)\right)$. Setting $\partial_{i}=\partial / \partial t_{i}$, we then have

$$
\left.\left(\partial_{i} \cdot \omega\right)\right|_{t=0}=-k \cdot \frac{s_{i} \omega(0)}{s_{0}^{k+1}}+\frac{\left.\partial_{i} \omega(t)\right|_{t=0}}{s_{0}^{k}} \equiv-k \cdot \frac{s_{i} \omega(0)}{s_{0}^{k+1}} \quad \bmod F_{k} \mathcal{N} .
$$

Thus surjectivity of (7.2) on stalks is equivalent to surjectivity of the product maps

$$
H^{0}\left(X, \mathscr{O}_{X}(1)\right) \otimes H^{0}\left(X, \Omega_{X}^{n}(k)\right) \rightarrow H^{0}\left(X, \Omega_{X}^{n}(k+1)\right),
$$

which is satisfied for $k \gg 0$ because $\mathscr{O}_{X}(1)$ is very ample.

Since it is illustrative, we shall also compute the characteristic variety of $\mathcal{N}$; more precisely, we shall compute the characteristic sheaf $\mathscr{C}(\mathcal{N}, F)$. To state the result, let $\phi: \mathscr{X} \rightarrow X$ be the projection to the second coordinate, and recall that $\mathscr{X}$ is naturally embedded into $\mathbb{P}\left(\Theta_{P}\right)$.

Lemma 7.3. We have $\mathscr{C}(\mathcal{N}, F) \simeq \phi^{*} \Omega_{X}^{n}$; consequently, the characteristic variety of $\mathcal{N}$ is the cone over $\mathscr{X}$, and thus has a single irreducible component of dimension $\operatorname{dim} \mathscr{X}+1=d+n$ and multiplicity one.

Proof. As explained in $\$ 5$ the coherent sheaf $\mathscr{C}(\mathcal{N}, F)$ is determined by its module of sections

$$
C(\mathcal{N}, F)=\bigoplus_{k \in \mathbb{Z}} H^{0}\left(P, F_{k} \mathcal{N} / F_{k-1} \mathcal{N}\right) .
$$

To compute the individual summands, recall that

$$
F_{k} \mathcal{N}=p r_{P *} \Omega_{P \times X / P}^{n}(k \mathscr{X}) \simeq H^{0}\left(X, \Omega_{X}^{n}(k)\right) \otimes \mathscr{O}_{P}(k)
$$

for $k \geq 1$ (and 0 otherwise). Thus $H^{1}\left(P, F_{k-1} \mathcal{N}\right)=0$, and so

$$
H^{0}\left(P, F_{k} \mathcal{N} / F_{k-1} \mathcal{N}\right) \simeq \frac{H^{0}\left(P, F_{k} \mathcal{N}\right)}{H^{0}\left(P, F_{k-1} \mathcal{N}\right)} \simeq \frac{H^{0}\left(P \times X, \Omega_{P \times X / P}^{n}(k \mathscr{X})\right)}{H^{0}\left(P \times X, \Omega_{P \times X / P}^{n}((k-1) \mathscr{X})\right)} .
$$

Writing $\mathscr{O}_{\mathscr{X}}(1)$ for the restriction of $\mathscr{O}_{P \times X}(\mathscr{X})$ to the universal hyperplane section, we have the exact sequence

$$
0 \longrightarrow \Omega_{P \times X / P}^{n}((k-1) \mathscr{X}) \longrightarrow \Omega_{P \times X / P}^{n}(k \mathscr{X}) \longrightarrow \phi^{*} \Omega_{X}^{n} \otimes \mathscr{O}_{\mathscr{X}}(k) \longrightarrow 0 .
$$

The higher cohomology of the first term being zero for $k \gg 0$, we thus get

$$
H^{0}\left(P, F_{k} \mathcal{N} / F_{k-1} \mathcal{N}\right) \simeq H^{0}\left(\mathscr{X}, \phi^{*} \Omega_{X}^{n} \otimes \mathscr{O}_{\mathscr{X}}(k)\right),
$$

and hence $C(\mathcal{N}, F)$ agrees in large degrees with the graded module

$$
\bigoplus_{k=0}^{\infty} H^{0}\left(\mathscr{X}, \phi^{*} \Omega_{X}^{n} \otimes \mathscr{O}_{\mathscr{X}}(k)\right)
$$

This means that the characteristic sheaf $\mathscr{C}(\mathcal{N}, F)$ is precisely $\phi^{*} \Omega_{X}^{n}$, viewed as a sheaf on $\mathbb{P}\left(\Theta_{P}\right)$ under the inclusion $\mathscr{X} \hookrightarrow \mathbb{P}\left(\Theta_{P}\right)$. 
8. The object of interest. We now define the $\mathcal{D}$-module that we are actually interested in. Let $\mathscr{H}_{\mathscr{O}}$ be the vector bundle

$$
\mathscr{H}_{\mathscr{O}}=\mathscr{O}_{P^{s m}} \otimes_{\mathbb{C}} R_{e v}^{n-1} \pi_{*}^{s m} \mathbb{C}
$$

with fibers $H_{e v}^{n-1}\left(\mathscr{X}_{p}, \mathbb{C}\right)$. It underlies the variation of Hodge structure $R_{e v}^{n-1} \pi_{*}^{s m} \mathbb{Q}$ on the vanishing cohomology of the hyperplane sections. Let $j: P^{s m} \hookrightarrow P$ be the inclusion. The operation of taking fiberwise residues defines a map of sheaves

$$
\operatorname{Res} \mathscr{X}_{P}: \mathcal{N} \rightarrow j_{*} \mathscr{H}_{\mathscr{O}}
$$

concretely, for $\omega \in \Gamma(U, \mathcal{N})$, we let $\operatorname{Res} \mathscr{X} / P(\omega) \in \Gamma\left(U \cap P^{s m}, \mathscr{H}_{\mathscr{O}}\right)$ be the section whose value at a point $p \in U \cap P^{s m}$ is

$$
\operatorname{Res} \mathscr{X}_{p}\left(\left.\omega\right|_{X \backslash \mathscr{X}_{p}}\right) \in H_{e v}^{n-1}\left(\mathscr{X}_{p}, \mathbb{C}\right) .
$$

We define $\mathcal{M}$ as the image sheaf, and also let $F_{k} \mathcal{M}$ be the image of $F_{k} \mathcal{N}$. Over $P^{s m}$, taking residue commutes with the action by vector fields [Voi02, p. 425-6], and we can therefore give $\mathcal{M}$ the induced $\mathcal{D}$-module structure. Then $F_{\bullet} \mathcal{M}$ is a good filtration on $\mathcal{M}$, and $\operatorname{Res}_{\mathscr{X} / P}: \mathcal{N} \rightarrow \mathcal{M}$ is a map of filtered $\mathcal{D}$-modules.

Clearly, $\operatorname{Res} \mathscr{X} / P$ is surjective over $P^{s m}$, and so we have $j^{*} \mathcal{M}=\mathscr{H}_{\mathscr{O}}$; this is consistent with the $\mathcal{D}$-module structure on $\mathscr{H}_{\mathscr{O}}$ given by the Gauss-Manin connection. Moreover, once $\mathscr{O}_{X}(1)$ is sufficiently ample, we even have

$$
j^{*} F_{k} \mathcal{M}=F^{n-k} \mathscr{H}_{\mathscr{O}} \text {. }
$$

because the Hodge filtration is determined by pole order. Thus $(\mathcal{M}, F)$ is an extension of $\left(\mathscr{H}_{\mathscr{O}}, F\right)$ to a filtered $\mathcal{D}$-module on $P$; we will see later that $\mathcal{M}$ is, in fact, the minimal extension in the category of holonomic $\mathcal{D}$-modules.

\section{Mixed Hodge modules}

In this section, we investigate the relationship between our filtered $\mathcal{D}$-module and M. Saito's theory of mixed Hodge modules; the main point is that, up to a shift in the filtration, $(\mathcal{M}, F)$ underlies a Hodge module.

9. Mixed Hodge modules. Instead of reviewing Saito's theory in the abstract, we shall concentrate on a specific example: how to prove the following familiar result using mixed Hodge modules.

Proposition 9.1. Let $X$ be a smooth complex projective variety of dimension n, and let $D \subseteq X$ be a smooth hypersurface. Assume that $\mathscr{O}_{X}(D)$ is ample enough so that $H^{q}\left(X, \Omega_{X}^{p}(k D)\right)=0$ for $q>0$ and $k>0$. Then the cohomology in degree $k$ of the complex

$$
H^{0}\left(X, \Omega_{X}^{p}(D)\right) \stackrel{d}{\rightarrow} H^{0}\left(X, \Omega_{X}^{p+1}(2 D)\right)-\cdots \rightarrow H^{0}\left(X, \Omega_{X}^{n}((n-p+1) D)\right)
$$

is isomorphic to $F^{p} H^{k}(X \backslash D, \mathbb{C})$. The isomorphism takes a d-closed rational form to the cohomology class defined by its restriction to $X \backslash D$.

For $X$ any quasi-projective complex algebraic variety, $\operatorname{MHM}(X)$ denotes the abelian category of algebraic mixed Hodge modules on $X$. Each mixed Hodge module $M$ has an underlying perverse sheaf $\operatorname{rat} M$, and the functor

$$
\text { rat: } \operatorname{MHM}(X) \rightarrow \operatorname{Perv}_{\text {alg }}(X)
$$

to the category of $\mathbb{Q}$-valued algebraic perverse sheaves on $X$ is fully faithful. (Here "algebraic" means that we only consider stratifications whose strata are algebraic 
varieties.) Each mixed Hodge module also has an underlying filtered holonomic $\mathcal{D}$-module $(\mathcal{M}, F)$, which corresponds to (the complexification of) rat $M$ under the Riemann-Hilbert correspondence; the isomorphism rat $M \otimes_{\mathbb{Q}} \mathbb{C} \simeq \mathrm{DR}_{X}(\mathcal{M})$ should be seen as providing the $\mathcal{D}$-module $\mathcal{M}$ with a $\mathbb{Q}$-structure. In order for $M$ to be a mixed Hodge module, several very restrictive conditions have to be satisfied (for instance, existence of a weight filtration, admissibility, good behavior under nearby and vanishing cycle functors, to name just a few); since we do not need them here, we refer to [Sai88, §4] for details.

The basic examples of mixed Hodge modules are (admissible, graded-polarized) variations of mixed Hodge structure. In the case of a point, $\operatorname{MHM}(p t)$ is the category of (graded-polarized) mixed Hodge structures defined over $\mathbb{Q}$. Following Saito, we let $\mathbb{Q}^{H} \in \operatorname{MHM}(p t)$ be the unique Hodge structure on $\mathbb{Q}$ of weight zero. With $d_{X}=\operatorname{dim} X$ and $a_{X}: X \rightarrow p t$ the structure map, we also define $\mathbb{Q}_{X}^{H}=$ $a_{X}^{*} \mathbb{Q}^{H} \in D^{b} \operatorname{MHM}(X)$. When $X$ is smooth, $\mathbb{Q}_{X}^{H}\left[d_{X}\right]$ is a pure Hodge module on $X$ whose underlying perverse sheaf is $\mathbb{Q}_{X}\left[d_{X}\right]$; the corresponding $\mathcal{D}$-module is $\mathscr{O}_{X}$, with filtration

$$
F_{k} \mathscr{O}_{X}= \begin{cases}\mathscr{O}_{X} & \text { if } k \geq 0 \\ 0 & \text { otherwise }\end{cases}
$$

Now suppose that $X$ is a smooth projective variety of dimension $n$, and that $D \subseteq X$ is a smooth divisor. The cohomology of $U=X \backslash D$ is governed by the mixed Hodge module $j_{*} \mathbb{Q}_{U}^{H}[n]$ on $X$, where $j: U \hookrightarrow X$ is the inclusion map. Since $D$ has no singularities, the underlying filtered $\mathcal{D}$-module is easy to describe: it is the sheaf $\mathcal{M}_{U}=\mathscr{O}_{X}(* D)$ of rational functions with poles along $D$, and the filtration

$$
F_{k} \mathcal{M}_{U}= \begin{cases}\mathscr{O}_{X}((k+1) D) & \text { if } k \geq 0 \\ 0 & \text { otherwise }\end{cases}
$$

is given by pole order (see [Sai93, Corollary 4.3] for more details).

To compute the Hodge filtration on the cohomology groups $H^{i}(U, \mathbb{Q})$, we observe that, as mixed Hodge structures,

$$
H^{i}(U) \simeq H^{i-n} a_{U *} \mathbb{Q}_{U}^{H}[n] \simeq H^{i-n} a_{X *}\left(j_{*} \mathbb{Q}_{U}^{H}[n]\right) .
$$

The underlying vector space can be computed with the help of the de Rham complex of the $\mathcal{D}$-module $\mathcal{M}_{U}$; this is the complex

$$
\operatorname{DR}_{X}\left(\mathcal{M}_{U}\right)=\left[\mathcal{M}_{U} \stackrel{d}{\rightarrow} \Omega_{X}^{1} \otimes \mathcal{M}_{U}-\cdots \rightarrow \Omega_{X}^{n} \otimes \mathcal{M}_{U}\right][n]
$$

supported in degrees $-n, \ldots, 0$. Its hypercohomology group $\mathbb{H}^{k}\left(\operatorname{DR}_{X}\left(\mathcal{M}_{U}\right)\right)$ is the $\mathbb{C}$-vector space underlying the mixed Hodge structure $H^{k} a_{U *} \mathbb{Q}_{U}^{H}[n]$.

The de Rham complex is naturally filtered by the subcomplexes

$$
F_{k} \operatorname{DR}_{X}\left(\mathcal{M}_{U}\right)=\left[F_{k} \mathcal{M}_{U} \rightarrow \Omega_{X}^{1} \otimes F_{k+1} \mathcal{M}_{U}-\cdots \rightarrow \Omega_{X}^{n} \otimes F_{k+n} \mathcal{M}_{U}\right][n],
$$

and the induced filtration on hypercohomology, and hence on $H^{k} a_{U *} \mathbb{Q}_{U}^{H}[n]$, is exactly the Hodge filtration. Moreover, since $X$ is smooth and projective, the filtration is actually strict, meaning that the map $\mathbb{H}^{k}\left(F_{k} \operatorname{DR}_{X}\left(\mathcal{M}_{U}\right)\right) \rightarrow \mathbb{H}^{k}\left(\operatorname{DR}_{X}\left(\mathcal{M}_{U}\right)\right)$ is injective. It is now very easy to compute the Hodge filtration on $H^{i}(U, \mathbb{C})$. Indeed,

$$
F^{p} H^{i}(U, \mathbb{C}) \simeq F_{-p} \mathbb{H}^{i-n}\left(\mathrm{DR}_{X}\left(\mathcal{M}_{U}\right)\right) \simeq \mathbb{H}^{i-n}\left(F_{-p} \operatorname{DR}_{X}\left(\mathcal{M}_{U}\right)\right),
$$


and the term in degree $i-n$ of the indicated complex is

$$
\Omega_{X}^{n+(i-n)} \otimes F_{-p+n+(i-n)} \mathcal{M}_{U}= \begin{cases}\Omega_{X}^{i}((i-p+1) D) & \text { for } i \geq p, \\ 0 & \text { otherwise. }\end{cases}
$$

Under the ampleness assumptions on $D$, each of these sheaves has no higher cohomology, and so we obtain the assertion of Proposition 9.1.

10. Cohomology of the universal hypersurface. We take from [BFNP07] the idea of studying the universal hypersurface by means of mixed Hodge modules. Where convenient for the purpose of this paper, we reprove some of their results using the theory of $\mathcal{D}$-modules instead of perverse sheaves. For notational economy, let $d=\operatorname{dim} P, n=\operatorname{dim} X$, and $d_{\mathscr{X}}=n+d-1$.

The crucial point is that $\mathscr{X}$ is nonsingular; this makes it possible to use the Decomposition Theorem. Recall from [BFNP07, 4.5] that there is a decomposition

$$
\pi_{*} \mathbb{Q}_{\mathscr{X}}^{H}\left[d_{\mathscr{X}}\right] \simeq \bigoplus_{i, j} E_{i, j}[-i]
$$

in the derived category $D^{b} \operatorname{MHM}(P)$. Indeed, since $\mathscr{X}$ is smooth and projective, the mixed Hodge module $\mathbb{Q}_{\mathscr{C}}^{H}\left[d_{\mathscr{X}}\right]$ on $\mathscr{X}$ is pure of weight $d_{\mathscr{X}}$; the same is then true for its image under $\pi_{*}$ because $\pi$ is smooth and projective. Applying Saito's version of the Decomposition Theorem, we have

$$
\pi_{*} \mathbb{Q}_{\mathscr{X}}^{H}\left[d_{\mathscr{X}}\right] \simeq \bigoplus_{i} E_{i}[-i],
$$

where $E_{i}=H^{i} \pi_{*} \mathbb{Q}_{\mathscr{X}}^{H}\left[d_{\mathscr{X}}\right]$. We can further decompose each $E_{i}$ into simple Hodge modules; let $E_{i, j}$ be the direct sum of all those pieces whose codimension of strict support is equal to $j$. We then arrive at the isomorphism in (10.1), with $E_{i, j}$ pure of weight $d_{\mathscr{X}}+i$ and supported on a closed subscheme of $P$ of codimension $j$. Moreover, we see that

$$
H^{i} \pi_{*} \mathbb{Q}_{\mathscr{X}}^{H}\left[d_{\mathscr{X}}\right] \simeq \bigoplus_{j=0}^{d} E_{i, j}
$$

for all $i \in \mathbb{Z}$. This decomposition is most interesting when $i=0$.

One general result about the decomposition is Saito's Hard Lefschetz Theorem Sai90, Théorème 1 on p. 853], which asserts that

$$
E_{i, j} \simeq E_{-i, j}(-i) ;
$$

note that it again depends on the fact that $\pi$ is projective and smooth. Essentially all the individual Hodge modules $E_{i, j}$ have been computed in [BFNP07; for the convenience of the reader, we summarize the main results:

(a) Perverse Weak Lefschetz Theorem: $E_{i, j}=0$ unless $i=0$ or $j=0$.

(b) $E_{i, 0} \simeq H^{n+i-1}(X) \otimes \mathbb{Q}_{P}^{H}[d]$ for $i<0$.

(c) $E_{0,0} \simeq j_{! *} V^{n-1} \simeq j_{! *} V_{e v}^{n-1} \oplus H^{n-1}(X) \otimes \mathbb{Q}_{P}^{H}[d]$.

(d) $E_{0,1}=0$ iff the vanishing cohomology $V_{e v}^{n-1}$ is nonzero.

(e) $E_{0, j}=0$ for all $j>0$, provided that $\mathscr{O}_{X}(1)$ is sufficiently ample.

To understand the notation, recall that $j: P^{s m} \hookrightarrow P$ is the inclusion of the open set where the map $\pi: \mathscr{X} \rightarrow P$ is smooth. Let $V^{n-1}=H^{n-1} \pi_{*}^{s m} \mathbb{Q}_{\mathscr{X}}^{H}[d]$ denote the 
Hodge module on $P^{s m}$ whose underlying perverse sheaf is the shifted local system $R^{n-1} \pi_{*}^{s m} \mathbb{Q}[d] ;$ it admits a direct sum decomposition

$$
V^{n-1}=V_{e v}^{n-1} \oplus H^{n-1}(X) \otimes \mathbb{Q}_{P}^{H}[d],
$$

where $V_{e v}^{n-1}$ is the polarized variation of Hodge structure given by the vanishing cohomology of the fibers, viewed as an element of $\operatorname{MHM}\left(P^{s m}\right)$. Finally, $j_{! *}$ is the intermediate extension functor.

The methods of this paper produce a stronger vanishing theorem for the modules $E_{0, j}$, namely that $E_{0, j}=0$ for every $j \geq 2$ (the proof can be found in 915 below). If we combine this small improvement with the results of [BFNP07, we obtain the following useful formulas for the cohomology of the universal hypersurface.

Theorem 10.5 (Brosnan, Fang, Nie, and Pearlstein). If the vanishing cohomology of the hypersurfaces is nontrivial, then

$$
H^{k} \pi_{*} \mathbb{Q}_{\mathscr{X}}^{H}\left[d_{\mathscr{X}}\right] \simeq \begin{cases}H^{k+n-1}(X) \otimes \mathbb{Q}_{P}^{H}[d] & \text { for } k<0, \\ H^{k+n+1}(X)(1) \otimes \mathbb{Q}_{P}^{H}[d] & \text { for } k>0, \\ j_{!_{*}} V_{e v}^{n-1} \oplus H^{n-1}(X) \otimes \mathbb{Q}_{P}^{H}[d] & \text { for } k=0,\end{cases}
$$

as polarized Hodge modules on $P$ of weight $k+(d+n-1)$.

Proof. This follows from (10.2) and the other results quoted above.

11. An exact sequence. We shall now derive most of the above results from a somewhat different point of view; this will also show how the $\mathcal{D}$-module $(\mathcal{M}, F)$ is connected to mixed Hodge modules.

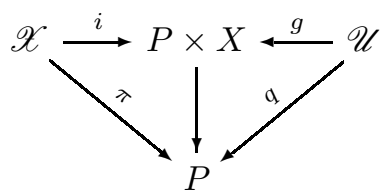

Let $\mathscr{U}=P \times X \backslash \mathscr{X}$ be the complement of the universal hypersurface. We denote by $g$ the inclusion of $\mathscr{U}$ into $P \times X$, and by $i$ that of $\mathscr{X}$ into $P \times X$, as shown in the diagram. Our starting point is the distinguished triangle

$$
i_{*} i^{!} \mathbb{Q}_{P \times X}^{H} \rightarrow \mathbb{Q}_{P \times X}^{H} \rightarrow g_{*} g^{*} \mathbb{Q}_{P \times X}^{H} \longrightarrow i_{*} i^{!} \mathbb{Q}_{P \times X}^{H}[1]
$$

in the category $D^{b} \operatorname{MHM}(P \times X)$ Sai90, (4.4.1) on p. 321]. Obviously, $g^{*} \mathbb{Q}_{P \times X}^{H}=$ $\mathbb{Q}_{\mathscr{U}}^{H}$; moreover, since $\mathscr{X} \subseteq P \times X$ is a smooth hypersurface, Verdier duality gives an isomorphism $i^{!} \mathbb{Q}_{P \times X}^{H} \simeq \mathbb{Q}_{\mathscr{X}}^{H}(-1)[-2]$. We can therefore rotate the triangle one step to the left and shift by $d_{P \times X}$ steps to obtain

$$
\mathbb{Q}_{P \times X}^{H}\left[d_{P \times X}\right] \rightarrow g_{*} \mathbb{Q}_{\mathscr{U}}^{H}\left[d_{\mathscr{U}}\right] \rightarrow i_{*} \mathbb{Q}_{\mathscr{X}}^{H}(-1)\left[d_{\mathscr{X}}\right] \rightarrow \mathbb{Q}_{P \times X}^{H}\left[d_{P \times X}+1\right]
$$


We then apply the functor $p r_{P *}$ and take cohomology; this gives a long exact sequence in $\operatorname{MHM}(P)$, a typical portion of which is (for $k \in \mathbb{Z}$ arbitrary)

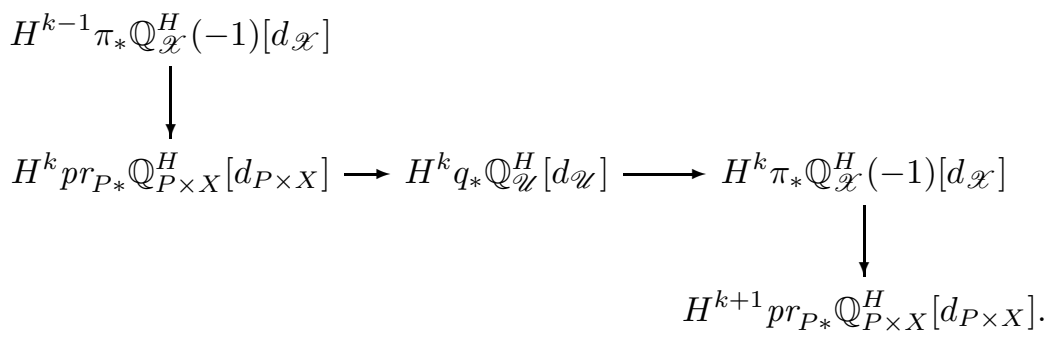

Ultimately, we would like to find $H^{0} \pi_{*} \mathbb{Q}_{\mathscr{C}}^{H}\left[d_{\mathscr{X}}\right]$; this makes it necessary to compute the other mixed Hodge modules in the sequence. Throughout, $H^{i}(X)$ denotes the Hodge structure on the $i$-th cohomology of $X$, viewed as an object in $\operatorname{MHM}(p t)$.

Lemma 11.2. For each $k \in \mathbb{Z}$, we have $H^{k} p r_{P *} \mathbb{Q}_{P \times X}^{H}\left[d_{P \times X}\right] \simeq H^{k+n}(X) \otimes \mathbb{Q}_{P}^{H}[d]$.

Proof. By Proper Base Change Sai90, (4.4.3) on p. 323] for the diagram

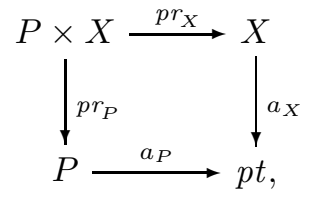

we have $p r_{P *} \mathbb{Q}_{P \times X}^{H}=p r_{P *} p r_{X}^{*} \mathbb{Q}_{X}^{H}=a_{P}^{*} a_{X *} \mathbb{Q}_{X}^{H}$. From the decomposition

$$
a_{X *} \mathbb{Q}_{X}^{H} \simeq \bigoplus_{i} H^{i} a_{X *} \mathbb{Q}_{X}^{H}[-i]=\bigoplus_{i} H^{i}(X)[-i]
$$

in $\operatorname{MHM}(p t)$ [Sai88, Corollaire 3 on p. 857], it follows that

$$
\operatorname{pr}_{P *} \mathbb{Q}_{P \times X}^{H}\left[d_{P \times X}\right] \simeq a_{P}^{*} \bigoplus_{i} H^{i}(X)\left[d_{P \times X}-i\right]=\bigoplus_{i} H^{i}(X) \otimes \mathbb{Q}_{P}^{H}\left[d_{P \times X}-i\right] .
$$

Now apply $H^{k}$ to get $H^{k} p r_{P *} \mathbb{Q}_{P \times X}^{H}\left[d_{P \times X}\right] \simeq H^{k+n}(X) \otimes \mathbb{Q}_{P}^{H}[d]$, since $\mathbb{Q}_{P}^{H}\left[d_{P \times X}-i\right]$ sits in degree $d-\left(d_{P \times X}-i\right)=i-n$.

The cohomology of a smooth affine variety vanishes above the middle dimension. The following lemma shows that a similar result is true for the cohomology of $q_{*} \mathbb{Q}_{\mathscr{U}}^{H}\left[d_{\mathscr{U}}\right]$; given that $U=P \times X \backslash \mathscr{X}$ is affine, this is not surprising.

Lemma 11.3. We have $H^{k} q_{*} \mathbb{Q}_{\mathscr{U}}^{H}\left[d_{\mathscr{U}}\right]=0$ for all $k>0$.

Proof. We give a proof using $\mathcal{D}$-modules. The mixed Hodge module $g_{*} \mathbb{Q}_{\mathscr{U}}^{H}\left[d_{\mathscr{U}}\right]$ has underlying $\mathcal{D}$-module $\mathscr{O}_{P \times X}(* \mathscr{X})$, because $\mathscr{X}$ is nonsingular [Sai93, Corollary 4.3]. The $\mathcal{D}$-module associated to $q_{*} \mathbb{Q}_{\mathscr{U}}^{H}\left[d_{\mathscr{U}}\right]=p r_{P *} g_{*} \mathbb{Q}_{\mathscr{U}}^{H}\left[d_{\mathscr{U}}\right]$ is therefore the direct image of $\mathscr{O}_{P \times X}(* \mathscr{X})$; it can be computed from the relative de Rham complex

$$
\begin{aligned}
\mathrm{DR}_{P \times X / P}\left(\mathscr{O}_{P \times X}(* \mathscr{X})\right)= \\
\quad\left[\mathscr{O}_{P \times X}(* \mathscr{X}) \rightarrow \Omega_{P \times X / P}^{1}(* \mathscr{X})-\cdots \rightarrow \Omega_{P \times X / P}^{n}(* \mathscr{X})\right][n] .
\end{aligned}
$$

Noting that each sheaf in the complex is acyclic for the functor $p r_{P *}$, the direct image is represented by the complex $\operatorname{pr}_{P *} \mathrm{DR}_{P \times X / P}\left(\mathscr{O}_{P \times X}(* \mathscr{X})\right)$. It is clearly supported in degrees $-n, \ldots, 0$, and therefore the cohomology sheaf in degree $k>0$ 
vanishes. Since the functor that takes a mixed Hodge module to its underlying $\mathcal{D}$ module is faithful, we conclude that $H^{k} q_{*} \mathbb{Q}_{\mathscr{U}}^{H}\left[d_{\mathscr{U}}\right]$ is also zero.

Finally, we borrow the following lemma from [BFNP07, Proposition 4.8].

Lemma 11.4 (Brosnan, Fang, Nie, and Pearlstein). We have

$$
E_{0,0} \simeq j_{! *} V^{n-1} \simeq j_{! *} V_{e v}^{n-1} \oplus H^{n-1}(X) \otimes \mathbb{Q}_{P}^{H}[d] .
$$

Proof. By definition, $E_{0,0}$ is the piece in the decomposition of $H^{0} \pi_{*} \mathbb{Q}_{\mathscr{X}}^{H}\left[d_{\mathscr{X}}\right]$ that has strict support equal to all of $P$. By the Base Change Theorem, applied to the inclusion $j: P^{s m} \rightarrow P$, we have

$$
j^{*} H^{0} \pi_{*} \mathbb{Q}_{\mathscr{X}}^{H}\left[d_{\mathscr{X}}\right] \simeq H^{0} \pi_{*}^{s m} \mathbb{Q}_{\mathscr{X}^{s m}}^{H}\left[d_{\mathscr{X}}\right]=H^{n-1} \pi_{*}^{s m} \mathbb{Q}_{\mathscr{X}}^{H}[d]=V^{n-1} .
$$

Therefore, $j_{! *} V^{n-1}$ is a submodule of $H^{0} \pi_{*} \mathbb{Q}_{\mathscr{X}}^{H}\left[d_{\mathscr{X}}\right]$. Since all other terms $E_{0, j}$ are supported in proper subvarieties, we conclude that $j_{!_{*}} V^{n-1} \simeq E_{0,0}$. The second isomorphism is then an immediate consequence of (10.4).

12. Analysis of the exact sequence. We can now look at the exact sequence in (11.1) one more time. For $k>0$, each portion of the sequence simplifies to

$$
0 \longrightarrow H^{k} \pi_{*} \mathbb{Q}_{\mathscr{X}}^{H}(-1)\left[d_{\mathscr{X}}\right] \longrightarrow H^{n+1+k}(X) \otimes \mathbb{Q}_{P}^{H}[d] \longrightarrow 0,
$$

using the vanishing in Lemma 11.3, and the result of Lemma 11.2, In terms of the decomposition (10.2), we thus have

$$
E_{k, 0}=E_{k} \simeq H^{n+1+k}(X)(1) \otimes \mathbb{Q}_{P}^{H}[d] \text { for } k>0,
$$

noting that $E_{k, j}=0$ if $j \neq 0$ because $E_{k}$ is supported on all of $P$. For $k<0$, we deduce from this and Saito's Hard Lefschetz Theorem (10.3) that

$$
E_{k, 0} \simeq E_{-k, 0}(-k) \simeq H^{n+1-k}(X)(1-k) \otimes \mathbb{Q}_{P}^{H}[d] \simeq H^{n+k-1}(X) \otimes \mathbb{Q}_{P}^{H}[d],
$$

where the last isomorphism is because of the usual Hard Lefschetz Theorem. Since both isomorphisms are induced by the polarization, and therefore compatible, it follows that the restriction map $H^{n+k-1}(X) \otimes \mathbb{Q}_{P}^{H}[d] \rightarrow E_{k, 0}$ itself has to be an isomorphism. Again, $E_{k, j}=0$ if $k<0$ and $j \neq 0$, and so we have proved (a).

Next, we look at the exact sequence in negative degrees. After incorporating the results from above, a typical portion simplifies to

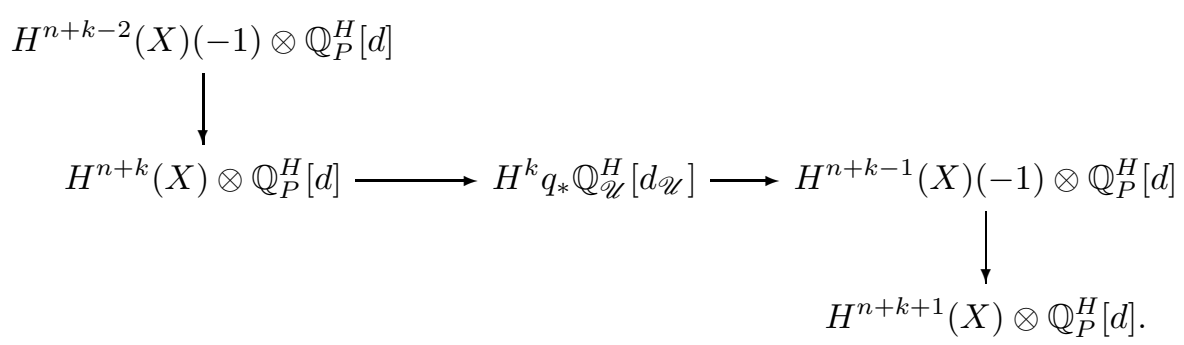

Both vertical maps are injective (by the usual Hard Lefschetz Theorem), and so we find that

$$
H^{k} q_{*} \mathbb{Q}_{\mathscr{U}}^{H}\left[d_{\mathscr{U}}\right] \simeq \frac{H^{n+k}(X)}{H^{n+k-2}(X)(-1)} \otimes \mathbb{Q}_{P}^{H}[d]
$$

when $k<0$. 
Finally, the part of the exact sequence for $k=0$ reads

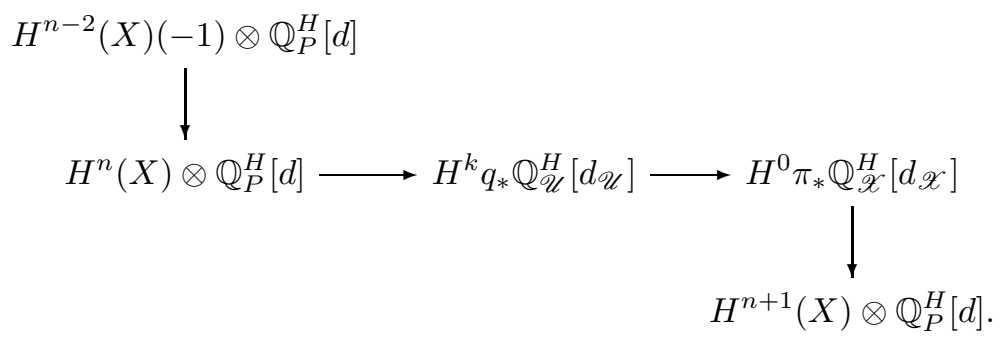

From (10.2) and Lemma 11.4, we have the decomposition

$$
H^{0} \pi_{*} \mathbb{Q}_{\mathscr{X}}^{H}\left[d_{\mathscr{X}}\right] \simeq j_{! *} V_{e v}^{n-1} \oplus H^{n-1}(X) \otimes \mathbb{Q}_{P}^{H}[d] \oplus \bigoplus_{j>0} E_{0, j} .
$$

Combining this with the fact that the primitive cohomology of weight $n$ satisfies $H_{0}^{n}(X) \simeq H^{n}(X) / H^{n-2}(X)(-1)$, we obtain the short exact sequence

$$
0 \longrightarrow H_{0}^{n}(X) \otimes \mathbb{Q}_{P}^{H}[d] \longrightarrow H^{0} q_{*} \mathbb{Q}_{\mathscr{U}}^{H}\left[d_{\mathscr{U}}\right] \longrightarrow j_{! *} V_{e v}^{n-1}(-1) \oplus R \longrightarrow 0
$$

Here $R=\bigoplus_{j>0} E_{0, j}(-1)$ is a sort of "error term," containing those pieces in the decomposition of $H^{0} \pi_{*} \mathbb{Q}_{\mathscr{X}}^{H}\left[d_{\mathscr{X}}\right]$ whose support is contained in the dual variety $X^{\vee}$. As mentioned above, it was shown in [BFNP07] that $R=0$ for sufficiently ample $\mathscr{O}_{X}(1)$; we give a more precise statement in 15 .

13. The underlying $\mathcal{D}$-modules. We now pass to the underlying $\mathcal{D}$-modules in the exact sequence (11.1). As explained during the proof of Lemma 11.3, the mixed Hodge module $g_{*} \mathbb{Q}_{\mathscr{U}}^{H}\left[d_{\mathscr{U}}\right]$ has associated $\mathcal{D}$-module $\mathscr{O}_{P \times X}(* \mathscr{X})$, with filtration

$$
F_{k} \mathscr{O}_{P \times X}(* \mathscr{X})= \begin{cases}\mathscr{O}_{P \times X}((k+1) \mathscr{X}) & \text { if } k \geq 0, \\ 0 & \text { otherwise. }\end{cases}
$$

To compute the direct images under the projection $P \times X \rightarrow P$, we use the relative de Rham complex

$$
\mathrm{DR}_{P \times X / P}=\left[\mathscr{O}_{P \times X} \rightarrow \Omega_{P \times X / P}^{1}-\cdots \rightarrow \Omega_{P \times X / P}^{n}\right][n],
$$

with differential $d_{P \times X / P}$. For $q>0$, we have

$$
R^{q} p r_{P *} \Omega_{P \times X / P}^{k} \otimes \mathscr{O}_{P \times X}(* \mathscr{X})=R^{q} p r_{P *} \Omega_{P \times X / P}^{k}(* \mathscr{X})=0,
$$

and so each of the sheaves $\Omega_{P \times X / P}^{k}(* \mathscr{X})$ is acyclic for the push-forward map $p r_{P *}$. Thus the complex $p r_{P *} \mathrm{DR}_{P \times X / P}\left(\mathscr{O}_{P \times X}(* \mathscr{X})\right)$, which looks like

$$
\left[p r_{P *} \mathscr{O}_{P \times X}(* \mathscr{X}) \rightarrow p r_{P *} \Omega_{P \times X / P}^{1}(* \mathscr{X})-\cdots \rightarrow p r_{P *} \Omega_{P \times X / P}^{n}(* \mathscr{X})\right][n],
$$

represents the direct image of $\mathscr{O}_{P \times X}(* \mathscr{X})$ in the derived category of filtered holonomic complexes on $P$. Note that each term in the complex is naturally a $\mathcal{D}$-module on $P$; moreover, the maps in the complex are $\mathscr{O}_{P}$-linear. We conclude that the $j$-th cohomology sheaf $\mathcal{N}^{j}$ of the complex,

$$
\mathcal{N}^{j}=H^{j} p r_{P *} \operatorname{DR}_{P \times X / P}\left(\mathscr{O}_{P \times X}(* \mathscr{X})\right),
$$

is the holonomic $\mathcal{D}$-module underlying the mixed Hodge module $H^{j} q_{*} \mathbb{Q}_{\mathscr{U}}^{H}\left[d_{\mathscr{U}}\right]=$ $H^{j} p r_{P *} g_{*} \mathbb{Q}_{\mathscr{U}}^{H}\left[d_{\mathscr{U}}\right]$. As in Lemma 11.3. $\mathcal{N}^{j}=0$ outside the range $-n \leq j \leq 0$. 
Both the complex in (13.2) and its cohomology sheaves $\mathcal{N}^{j}$ are naturally filtered. Indeed, the pole-order filtration on $\mathscr{O}_{P \times X}(* \mathscr{X})$ defines a filtration on the relative de Rham complex by subcomplexes $F_{k} \mathrm{DR}_{P \times X / P}\left(\mathscr{O}_{P \times X}(* \mathscr{X})\right)$, equal to

$$
\left[F_{k} \longrightarrow \Omega_{P \times X / P}^{1} \otimes F_{k+1}-\cdots \rightarrow \Omega_{P \times X / P}^{n} \otimes F_{k+n}\right][n],
$$

where $F_{k}=F_{k} \mathscr{O}_{P \times X}(* \mathscr{X})$ is as in (13.1). The filtration on the complex in (13.2) is then induced by the subcomplex $\mathbb{R} p r_{P *} F_{k} \mathrm{DR}_{P \times X / P}\left(\mathscr{O}_{P \times X}(* \mathscr{X})\right)$; note that $p r_{P *}$ means the derived functor here, as the individual sheaves in the complex are not necessarily acyclic. Since the morphism $p r_{P}$ is projective, the filtration is strict by a result of Saito's [Sai90, Theorem 2.14]; this means that the cohomology sheaves of the subcomplex inject into those of the whole complex. The filtration on each of the $\mathcal{D}$-modules $\mathcal{N}^{j}$ is the induced filtration,

$$
F_{k} \mathcal{N}^{j}=H^{j} p_{P *} F_{k} \operatorname{DR}_{P \times X / P}\left(\mathscr{O}_{P \times X}(* \mathscr{X})\right),
$$

where $p r_{P *}$ is again the derived functor. Observe that each filtered $\mathcal{D}$-module $\left(\mathcal{N}^{j}, F\right)$ is regular holonomic, because it underlies a mixed Hodge module.

Proposition 13.3. Let $F_{k}=F_{k} \mathscr{O}_{P \times X}(* \mathscr{X})$, and consider the subcomplex

$$
\left[p r_{P *} F_{k} \rightarrow p r_{P *} \Omega_{P \times X / P}^{1} \otimes F_{k+1}-\cdots \rightarrow p r_{P *} \Omega_{P \times X / P}^{n} \otimes F_{k+n}\right][n]
$$

of the complex in (13.2). Then for $k \gg 0$, the coherent sheaf $F_{k} \mathcal{N}^{j}$ is the $j$-th cohomology sheaf of the complex in (13.4). The same is true for arbitrary $k \in \mathbb{Z}$, provided that the line bundle $\mathscr{O}_{X}(1)$ satisfies the condition

$$
H^{q}\left(X, \Omega_{X}^{p}(k)\right)=0 \text { for } q>0 \text { and } k>0 .
$$

Proof. For $k \gg 0$, each sheaf in the complex is acyclic for the functor $p r_{P *}$. The same is true for arbitrary $k \in \mathbb{Z}$ under the displayed condition on $\mathscr{O}_{X}(1)$.

The most interesting among those $\mathcal{D}$-modules is

$$
\mathcal{N}^{0}=\frac{p r_{P *} \Omega_{P \times X / P}^{n}(* \mathscr{X})}{d_{P \times X / P}\left(p r_{P *} \Omega_{P \times X / P}^{n-1}(* \mathscr{X})\right)},
$$

which is filtered by the coherent subsheaves $F_{k} \mathcal{N}^{0}$. For $k \gg 0$, or in general if the condition in (13.5) is satisfied, these sheaves are given by the formula

$$
F_{k} \mathcal{N}^{0}=\frac{p r_{P *} \Omega_{P \times X / P}^{n}((n+k+1) \mathscr{X})}{\left.d_{P \times X / P}\left(p r_{P *} \Omega_{P \times X / P}^{n-1}((n+k) \mathscr{X})\right)\right)} .
$$

Now let $\left(\mathcal{M}_{e v}, F\right)$ be the filtered $\mathcal{D}$-module underlying $j_{! *} V_{e v}^{n-1}$; it is the minimal extension of the flat vector bundle $\mathscr{O}_{P s m} \otimes_{\mathbb{C}} R_{e v}^{n-1} \pi_{*}^{s m} \mathbb{C}$. Temporarily, we also introduce the filtered $\mathcal{D}$-module $(\mathcal{R}, F)$, underlying the error term $R=\bigoplus_{j>0} E_{0, j}$ in (12.3).

The following theorem summarizes the results of this and the previous section. To explain the notation, we remark that for a mixed Hodge structure $H$, the $\mathcal{D}$ module $H_{\mathbb{C}} \otimes \mathscr{O}_{P}$ underlying $a_{P}^{*} H=H \otimes \mathbb{Q}_{P}^{H}[d]$ has as its filtration

$$
F_{k}\left(H_{\mathbb{C}} \otimes \mathscr{O}_{P}\right)=\left(F_{k} H_{\mathbb{C}}\right) \otimes \mathscr{O}_{P}=\left(F^{-k} H_{\mathbb{C}}\right) \otimes \mathscr{O}_{P}
$$

induced from the Hodge filtration on $H$. 
Theorem 13.9. For any $j<0$, we have an isomorphism of filtered $\mathcal{D}$-modules

$$
\mathcal{N}^{j} \simeq \frac{H^{n+j}(X, \mathbb{C})}{H^{n+j-2}(X, \mathbb{C})} \otimes \mathscr{O}_{P},
$$

where the right-hand side has the filtration described in (13.8). Moreover, we have a short exact sequence of filtered $\mathcal{D}$-modules

$$
0 \longrightarrow H_{0}^{n}(X, \mathbb{C}) \otimes \mathscr{O}_{P} \longrightarrow \mathcal{N}^{0} \longrightarrow \mathcal{M}_{e v}(-1) \oplus \mathcal{R} \longrightarrow 0,
$$

strict with respect to the filtrations.

Proof. The first assertion is an immediate consequence of the isomorphism in (12.1). Indeed, we have just shown that the filtered $\mathcal{D}$-module underlying the left-hand side of (12.1) is $\mathcal{N}^{j}$, whereas the one underlying the right-hand side is evidently $H^{n+j}(X, \mathbb{C}) / H^{n+j-2}(X, \mathbb{C}) \otimes \mathscr{O}_{P}$. The second assertion follows in the same way from the exact sequence in (12.3), recalling that morphisms between mixed Hodge modules strictly preserve the Hodge filtrations on the underlying $\mathcal{D}$-modules.

In 15 , we show $R=0$ if and only if the vanishing cohomology of the hypersurfaces is nontrivial; this means that precisely one of $\mathcal{R}$ and $\mathcal{M}_{e v}$ is nonzero. After incorporating this result, we arrive at the following description of the filtered $\mathcal{D}$-module $\mathcal{M}$.

Corollary 13.10. Suppose that the vanishing cohomology of the hypersurfaces is nontrivial; then we have $\mathcal{M}_{e v}=\mathcal{M}$. If moreover the condition in (13.5) is satisfied, then $F_{k} \mathcal{M}_{e v}=F_{k+n} \mathcal{M}$, and the exact sequence in Theorem 13.9 becomes

$$
0 \longrightarrow H_{0}^{n}(X, \mathbb{C}) \otimes \mathscr{O}_{P} \longrightarrow \mathcal{N}^{0} \longrightarrow \mathcal{M}(-n-1) \longrightarrow 0 .
$$

Proof. By construction, the restriction of $\mathcal{M}_{e v}$ to $P^{s m}$ is the flat vector bundle $\mathscr{H}_{\mathscr{O}}$, and so $\mathcal{M}_{e v}$ is a subsheaf of $j_{*} \mathscr{H}_{\mathscr{O}}$ by the definition of the intermediate extension functor. Since $\mathcal{R}=0$ by Proposition [15.1, the $\mathcal{D}$-module $\mathcal{M}_{e v}$ is a quotient of $\mathcal{N}^{0}$, and over $P^{s m}$, the resulting map from $\left.\mathcal{N}^{0}\right|_{P^{s m}}$ to $\mathscr{H}_{\mathscr{O}}$ is clearly the fiberwise residue map. We conclude that $\mathcal{M}_{e v}=\mathcal{M}$. As for the filtrations, note that $F_{k} \mathcal{M}_{e v}$ is a quotient of $F_{k-1} \mathcal{N}^{0}$, and hence of $\operatorname{pr}_{P *} \Omega_{P \times X / P}^{n}((k+n) \mathscr{X})$.

14. Vanishing of higher cohomology. In this section, we use the direct image of the relative de Rham complex in (13.2) to construct something like a locally free resolution for the sheaves $F_{k} \mathcal{N}^{0}$, and then use it to prove a vanishing theorem for their higher cohomology groups. Fix $k \in \mathbb{Z}$; we assume either that (13.5) is satisfied, or that $k \gg 0$. Consider the subcomplex

$$
\mathcal{E}_{k}^{\bullet}=p r_{P *} F_{k-n-1} \operatorname{DR}_{P \times X / P}\left(\mathscr{O}_{P \times X}(* \mathscr{X})\right)
$$

of the direct image of the relative de Rham complex as in (13.4). It has $\mathscr{O}_{P}$-linear differentials, and is supported in degrees $-n, \ldots, 0$. The individual sheaves in the complex are easily found to be

$$
\begin{aligned}
\mathcal{E}_{k}^{i} & =p r_{P *}\left(\Omega_{P \times X / P}^{n+i} \otimes F_{k+i-1} \mathscr{O}_{P \times X}(* \mathscr{X})\right) \\
& = \begin{cases}H^{0}\left(X, \Omega_{X}^{n+i}(k+i)\right) \otimes \mathscr{O}_{P}(k+i) & \text { if } k+i \geq 1, \\
0 & \text { otherwise }\end{cases}
\end{aligned}
$$


The discussion in $\$ 13$ shows that the cohomology sheaves of the complex are precisely the coherent sheaves $F_{k-n-1} \mathcal{N}^{i}$. Theorem 13.9 describes these sheaves for $i<0$,

$$
F_{k-n-1} \mathcal{N}^{i} \simeq \frac{F^{n+1-k} H^{n+i}(X, \mathbb{C})}{F^{n-k} H^{n+i-2}(X, \mathbb{C})} \otimes \mathscr{O}_{P}
$$

This is almost as good as having a locally free resolution, and easily allows us to prove the following vanishing theorem.

Theorem 14.2. Suppose that the line bundle $\mathscr{O}_{X}(1)$ satisfies the condition in (13.5), or that $k \gg 0$. Then we have $H^{i}\left(P, \Omega_{P}^{p} \otimes F_{k} \mathcal{N}^{0}\right)=0$ for every $i \geq$ $\max (p-1,0)$.

Proof. The terms of the complex $\Omega_{P}^{p} \otimes \mathcal{E}_{k}^{\bullet}$ are direct sums of sheaves of the form $\Omega_{P}^{p}(m)$ with $m \geq 1$. In the first hypercohomology spectral sequence,

$$
{ }^{\prime} E_{1}^{i, j}=H^{j}\left(P, \Omega_{P}^{p} \otimes \mathcal{E}_{k}^{i}\right) \Longrightarrow \mathbb{H}^{i+j}\left(\Omega_{P}^{p} \otimes \mathcal{E}_{k}^{\bullet}\right),
$$

we have ' $E_{1}^{i, j}=0$ for $j>0$ by Bott's vanishing theorem. All but one row is therefore zero, and so the spectral sequence degenerates. Since moreover ${ }^{\prime} E_{1}^{i, 0}=0$ if $i>0$, it follows that $\mathbb{H}^{i}\left(\Omega_{P}^{p} \otimes \mathcal{E}_{k}^{\bullet}\right)=0$ whenever $i>0$.

To relate this to the cohomology of $F_{k-n-1} \mathcal{N}^{0}$, we use the second hypercohomology spectral sequence,

$$
{ }^{\prime \prime} E_{2}^{i, j}=H^{i}\left(P, \Omega_{P}^{p} \otimes F_{k-n-1} \mathcal{N}^{j}\right) \Longrightarrow \mathbb{H}^{i+j}\left(\Omega_{P}^{p} \otimes \mathcal{E}_{k}^{\bullet}\right),
$$

remembering that $F_{k-n-1} \mathcal{N}^{j}$ is the $j$-th cohomology sheaf of the complex $\mathcal{E}_{k}^{\bullet}$. Now $\Omega_{P}^{p} \otimes F_{k-n-1} \mathcal{N}^{j}$ is a direct sum of copies of $\Omega_{P}^{p}$ for $j<0$, and therefore ${ }^{\prime \prime} E_{2}^{i, j}=0$ unless $i=p$ or $j=0$. From the vanishing of the hypercohomology in positive degrees, we now conclude that

$$
H^{i}\left(P, \Omega_{P}^{p} \otimes F_{k-n-1} \mathcal{N}^{0}\right)={ }^{\prime \prime} E_{2}^{i, 0}=0
$$

for $i \geq \max (p-1,0)$, as asserted.

On the other hand, Corollary 13.10 relates $\mathcal{N}^{0}$ and $\mathcal{M}$ through the short exact sequence

$$
0 \longrightarrow F^{n+1-k} H_{0}^{n}(X, \mathbb{C}) \otimes \mathscr{O}_{P} \longrightarrow F_{k-n-1} \mathcal{N}^{0} \longrightarrow F_{k} \mathcal{M} \longrightarrow 0
$$

The following vanishing theorem for $F_{k} \mathcal{M}$ is an immediate consequence (note if the vanishing cohomology of the hypersurfaces is trivial, then $\mathcal{M}=0$, and so the result holds by default).

Corollary 14.3. If the condition in (13.5) is satisfied, then $H^{i}\left(P, \Omega_{P}^{p} \otimes F_{k} \mathcal{M}\right)=0$ for every $i \geq \max (p, 0)$.

The same spectral sequence argument can also be used to compute spaces of global sections. To state the result, we introduce the notation

$$
W_{k}^{p}=H^{0}\left(P \times X, \Omega_{P \times X / P}^{p}(k \mathscr{X})\right) \simeq H^{0}\left(X, \Omega_{X}^{p}(k)\right) \otimes H^{0}\left(P, \mathscr{O}_{P}(k)\right),
$$

noting that the relative differential $d_{P \times X / P}$ maps $W_{k}^{p}$ to $W_{k+1}^{p+1}$. 
Corollary 14.4. For $k \gg 0$, or for any $k \geq 1$ assuming (13.5), we have

$$
H^{0}\left(P, F_{k-n-1} \mathcal{N}^{0}\right) \simeq \frac{W_{k}^{n}}{d_{P \times X / P} W_{k-1}^{n-1}} .
$$

If the line bundle $\mathscr{O}_{X}(1)$ is sufficiently ample, then similarly

$$
H^{0}\left(P, F_{k} \mathcal{M}\right) \simeq \frac{W_{k}^{n}}{d_{P \times X / P} W_{k-1}^{n-1}+F^{n+1-k} H_{0}^{n}(X, \mathbb{C})} .
$$

Corollary 14.5. If $\mathscr{O}_{X}(1)$ satisfies the condition in (13.5), then $F_{k} \mathcal{M}$ is a quotient of the ample vector bundle $H^{0}\left(X, \Omega_{X}^{n}(k)\right) \otimes \mathscr{O}_{P}(k)$, and therefore globally generated.

Proof. $F_{k} \mathcal{M}$ is a quotient of $F_{k-n-1} \mathcal{N}^{0}$, which in turn is a quotient of $\mathcal{E}_{k}^{0}=$ $H^{0}\left(X, \Omega_{X}^{n}(k)\right) \otimes \mathscr{O}_{P}(k)$, at least when $k \geq 1$. This proves the assertion, because $F_{k} \mathcal{M}=0$ for $k \leq 0$.

15. The vanishing of the error term. Recall that the error term $R$ in (12.3) is the direct sum of the Hodge modules $E_{0, j}$ with $j>0$. In [BFNP07, it was shown that $R=0$ once the line bundle $\mathscr{O}_{X}(1)$ is sufficiently ample, and that $E_{0,1}=0$ precisely when the vanishing cohomology of the hypersurfaces is nontrivial. We improve this to a necessary and sufficient condition for the vanishing of the Hodge module $R$.

Proposition 15.1. We have $E_{0, j}=0$ for every $j \geq 2$. If the vanishing cohomology of the hypersurfaces is nontrivial, then $E_{0, j}=0$ for every $j>0$; in particular, the error term $R=0$ in (12.3) is then zero.

Note. By recent work of Dimca and Saito DS09, Theorem 6], it is known that the vanishing cohomology is nontrivial as soon as $\mathscr{O}_{X}(1)$ is the third (or, in most cases, second) power of a very ample line bundle.

The proof of the proposition is based on computing the characteristic variety of the $\mathcal{D}$-module $\mathcal{N}^{0}$; we will show that it has exactly two components, one of which is the cone over the set of singularities $\mathscr{Y} \subseteq \mathscr{X}$, and determine the multiplicities. Our starting point is the following simple lemma.

Lemma 15.2. As usual, let $\phi: \mathscr{X} \rightarrow X$ and $\psi: \mathscr{Y} \rightarrow X$ be the second projections. For every $k \in \mathbb{Z}$, the Koszul-type complex

$$
\mathscr{O}_{\mathscr{X}}(k-n) \rightarrow \phi^{*} \Omega_{X}^{1} \otimes \mathscr{O}_{\mathscr{X}}(k-n+1)-\cdots \rightarrow \phi^{*} \Omega_{X}^{n} \otimes \mathscr{O}_{\mathscr{X}}(k) .
$$

is a locally free resolution on $\mathscr{X}$ for the sheaf $\psi^{*} \Omega_{X}^{n} \otimes \mathscr{O}_{\mathscr{Y}}(k)$. The differential in this complex is given by the rule $\beta \mapsto d_{P \times X / P} s \mathscr{X} \wedge \beta$, where $s_{\mathscr{X}}$ is the section of $\mathscr{O}_{P \times X}(1)$ defining $\mathscr{X} \subseteq P \times X$.

Proof. From the embedding $i: X \hookrightarrow Q$ given by the linear system of $\mathscr{O}_{X}(1)$, we have the following exact sequence:

$$
0 \longrightarrow \Theta_{X} \longrightarrow i^{*} \Theta_{Q} \longrightarrow N_{X \mid Q} \longrightarrow 0 .
$$

The projectivization $\mathbb{P}\left(i^{*} \Theta_{Q}\right)$ is naturally isomorphic to $\mathscr{X}$, and the exact sequence determines a canonical section $\alpha_{\mathscr{X}}$ of the vector bundle $\left(\phi^{*} \Theta_{X}\right)^{\vee} \otimes \mathscr{O}_{\mathbb{P}\left(i^{*} \Theta_{Q}\right)}(1) \simeq$ $\phi^{*} \Omega_{X}^{1} \otimes \mathscr{O}_{\mathscr{X}}(1)$, whose zero scheme is $\mathbb{P}\left(N_{X \mid Q}\right) \simeq \mathscr{Y}$. As a consequence, $\mathscr{Y}$ is a locally complete intersection in $\mathscr{X}$, and its structure sheaf is resolved by the Koszul-type complex

$$
\mathscr{O}_{\mathscr{X}} \longrightarrow \phi^{*} \Omega_{X}^{1} \otimes \mathscr{O}_{\mathscr{X}}(1)-\cdots \rightarrow \phi^{*} \Omega_{X}^{n-1} \otimes \mathscr{O}_{\mathscr{X}}(n-1) \rightarrow \phi^{*} \Omega_{X}^{n} \otimes \mathscr{O}_{\mathscr{X}}(n),
$$


whose differential is given by wedge product with $\alpha_{\mathscr{X}}$. It is then not hard to see that $\alpha_{\mathscr{X}}=d_{P \times X / P} s \mathscr{X}$, viewed as a map

$$
d_{P \times X / P} s \mathscr{X}:\left.\mathscr{O}_{\mathscr{X}}(-1) \rightarrow \Omega_{P \times X / P}^{1}\right|_{\mathscr{X}} \simeq \phi^{*} \Omega_{X}^{1} .
$$

We obtain the asserted resolution after tensoring by $\mathscr{O}_{\mathscr{X}}(k-n)$.

We are now in a position to compute the coherent sheaf on $\mathbb{P}\left(\Theta_{P}\right)$ associated to the graded module $\mathrm{Gr}^{F} \mathcal{N}^{0}$.

Lemma 15.3. We have $\mathscr{C}\left(\mathcal{N}^{0}, F\right) \simeq \psi^{*} \Omega_{X}^{n} \otimes \mathscr{O}_{\mathscr{Y}}(n+1)$, viewed as a coherent sheaf on $\mathscr{Y} \hookrightarrow \mathbb{P}\left(\Theta_{P}\right)$ under the natural inclusion map.

Proof. As in Lemma 7.3, we shall compute the characteristic module $C\left(\mathcal{N}^{0}, F\right)$ directly, at least in sufficiently high degrees $k \gg 0$. To simplify the notation, we again use the notation

$$
W_{k}^{p}=H^{0}\left(P \times X, \Omega_{P \times X / P}^{p}(k \mathscr{X})\right) \simeq H^{0}\left(X, \Omega_{X}^{p}(k)\right) \otimes H^{0}\left(P, \mathscr{O}_{P}(k)\right)
$$

for the space of sections of the indicated vector bundle. By definition,

$$
C\left(\mathcal{N}^{0}, F\right)=\bigoplus_{k \in \mathbb{Z}} H^{0}\left(P, F_{k} \mathcal{N}^{0} / F_{k-1} \mathcal{N}^{0}\right),
$$

and since $H^{1}\left(P, F_{k} \mathcal{N}^{0}\right)=0$ for $k \gg 0$ by Theorem 14.2, we get that

$$
H^{0}\left(P, F_{k} \mathcal{N}^{0} / F_{k-1} \mathcal{N}^{0}\right) \simeq \frac{H^{0}\left(P, F_{k} \mathcal{N}^{0}\right)}{H^{0}\left(P, F_{k-1} \mathcal{N}^{0}\right)} .
$$

By Corollary 14.4, $H^{0}\left(P, F_{k} \mathcal{N}^{0}\right) \simeq W_{k+n+1}^{n} / d_{P \times X / P} W_{k+n}^{n-1}$, and so we finally have

$$
H^{0}\left(P, F_{k} \mathcal{N}^{0} / F_{k-1} \mathcal{N}^{0}\right) \simeq \frac{W_{k+n+1}^{n}}{d_{P \times X / P} W_{k+n}^{n-1}+W_{k+n}^{n}} .
$$

Next, we determine the graded module corresponding to $\psi^{*} \Omega_{X}^{n}$, given by

$$
\bigoplus_{k \in \mathbb{Z}} H^{0}\left(\mathscr{X}, \psi^{*} \Omega_{X}^{n} \otimes \mathscr{O}_{\mathscr{X}}(k)\right) \simeq \bigoplus_{k \in \mathbb{Z}} H^{0}\left(\mathscr{Y}, \psi^{*} \Omega_{X}^{n} \otimes \mathscr{O}_{\mathscr{Y}}(k)\right) .
$$

From Lemma 15.2, we know that the sequence

$$
\phi^{*} \Omega_{X}^{n-1} \otimes \mathscr{O}_{\mathscr{X}}(k-1) \longrightarrow \phi^{*} \Omega_{X}^{n} \otimes \mathscr{O}_{\mathscr{X}}(k) \longrightarrow \psi^{*} \Omega_{X}^{n} \otimes \mathscr{O}_{\mathscr{Y}}(k) \longrightarrow 0
$$

is exact; as $\mathscr{O}_{\mathscr{X}}(1)$ is ample, the corresponding sequence of global sections remains exact for $k \gg 0$. We therefore obtain

$$
H^{0}\left(\mathscr{Y}, \psi^{*} \Omega_{X}^{n} \otimes \mathscr{O}_{\mathscr{Y}}(k)\right) \simeq \frac{H^{0}\left(\mathscr{X}, \phi^{*} \Omega_{X}^{n} \otimes \mathscr{O}_{\mathscr{X}}(k)\right)}{H^{0}\left(\mathscr{X}, \phi^{*} \Omega_{X}^{n-1} \otimes \mathscr{O}_{\mathscr{X}}(k-1)\right)} .
$$

When $k \gg 0$, one easily shows that $H^{0}\left(\mathscr{X}, \phi^{*} \Omega_{X}^{p} \otimes \mathscr{O}_{\mathscr{X}}(k)\right) \simeq W_{k}^{p} / W_{k-1}^{p}$; since the differential in the exact sequence is given by $d_{P \times X / P} s \mathscr{X}$, we conclude that

$$
H^{0}\left(\mathscr{X}, \psi^{*} \Omega_{X}^{n} \otimes \mathscr{O}_{\mathscr{X}}(k)\right) \simeq \frac{W_{k}^{n}}{d_{P \times X / P} W_{k-1}^{n-1}+W_{k-1}^{n}}
$$

once $k$ is sufficiently large. The assertion now follows easily upon comparing the expressions in (15.4) and (15.5).

Now we turn to the proof of Proposition 15.1 
Proof. By Lemma 15.3, the characteristic sheaf $\mathscr{C}=\mathscr{C}\left(\mathcal{N}^{0}, F\right)$ is supported on $\mathscr{Y}$ and has rank one. Since the restriction of $\mathcal{N}^{0}$ to $P^{s m}$ is locally free, it follows that the characteristic variety of $\mathcal{N}^{0}$ has two irreducible components:

(1) The zero section of $T_{P}^{*}$, with multiplicity equal to the generic rank of $\mathcal{N}^{0}$.

(2) The cone over the set $\mathscr{Y}$, with multiplicity one.

The same is then true (with a different multiplicity for the first component) for the holonomic $\mathcal{D}$-module $\mathcal{M}_{e v} \oplus \mathcal{R}$, since it differs from $\mathcal{N}^{0}$ only by the vector bundle $H_{0}^{n}(X, \mathbb{C}) \otimes \mathscr{O}_{P}$ by Theorem 13.9. It follows that $E_{0, j}=0$ for $j \geq 2$ : indeed, the support of $E_{0, j}$ has codimension $j$ in $P$, and so its characteristic variety could not be contained in that of $\mathcal{N}^{0}$ unless $j=1$.

Now suppose that the vanishing cohomology of the hypersurfaces is nontrivial. Multiplicity is an additive function on holonomic $\mathcal{D}$-modules, and so one of $\mathcal{M}_{e v}$ and $\mathcal{R}$ has to have multiplicity zero along the cone over $\mathscr{Y}$. Since $\mathcal{R}$ is already supported inside $X^{\vee}$, we conclude that if $\mathcal{R} \neq 0$, the characteristic variety of $\mathcal{M}_{e v}$ has to consist of just the zero section, which means that $\mathcal{M}_{e v}$ has to be a locally free sheaf. But this can only happen when $\mathcal{M}_{e v}=0$, because the monodromy action on the vanishing cohomology is irreducible [Voi02, Corollaire 15.28]. Indeed, if the $\mathcal{D}$-module $\mathcal{M}_{e v}$ was locally free, it would be a flat vector bundle, and therefore trivial (because $P$ is simply connected). In particular, the local system $R_{e v}^{n-1} \pi_{*}^{s m} \mathbb{C}$ would be trivial. But since it is known that $H^{0}\left(P^{s m}, R_{e v}^{n-1} \pi_{*}^{s m} \mathbb{C}\right)=0$, this is not possible unless $R_{e v}^{n-1} \pi_{*}^{s m} \mathbb{C}=0$. Our assumptions rule out this possibility, and we conclude that $\mathcal{M}_{e v} \neq 0$ and hence $\mathcal{R}=0$.

Example 15.6. It is illustrative to compare our result with the example of plane conics, given in BFNP07, Example 5.13]. This is the special case when $X=\mathbb{P}^{2}$, with line bundle $\mathscr{O}_{\mathbb{P}^{2}}(2)$. As the authors remark, $E_{0,0}=0$, and $E_{0, j}=0$ for all $j \geq 2$, but $E_{0,1} \neq 0$. In other words, $\mathcal{M}_{e v}=0$ while $\mathcal{R} \neq 0$; note that only one of the summands of $\mathcal{R}$ is nonzero, as required by the multiplicity calculation above.

We conclude with a description of the characteristic sheaf of $(\mathcal{M}, F)$.

Corollary 15.7. If the vanishing cohomology of the hypersurfaces is nontrivial, we have $\mathscr{C}(\mathcal{M}, F) \simeq \psi^{*} \Omega_{X}^{n}$.

Proof. This follows almost immediately from the calculations just done. Indeed, consider the short exact sequence in Corollary 13.10. It shows that $\mathcal{M}$ and $\mathcal{N}^{0}$ differ only by the locally free sheaf $H_{0}^{n}(X, \mathbb{C}) \otimes \mathscr{O}_{P}$, and so we have the isomorphism

$$
\mathrm{Gr}_{k}^{F} \mathcal{M} \simeq \mathrm{Gr}_{k-n-1}^{F} \mathcal{N}^{0}
$$

it holds for any $k>0$ provided that (13.5) is satisfied, and in general for $k \gg 0$, which is sufficient here. We then get $\mathscr{C}(\mathcal{M}, F) \simeq \psi^{*} \Omega_{X}^{n}$ from Lemma 15.3 .

16. Hypercohomology of the de Rham complex. In this section, we assume that the vanishing cohomology of the hypersurfaces is nontrivial, so that $\mathcal{M}_{e v}=\mathcal{M}$ by Corollary 13.10, The $\mathcal{D}$-module $\mathcal{M}$ then underlies the Hodge module $j_{! *} V_{e v}^{n-1}$ on $P$. On the other hand, the corresponding perverse sheaf is

$$
\text { rat } j_{! *} V_{e v}^{n-1}=j_{! *} \operatorname{rat} V_{e v}^{n-1}=j_{!_{*}} R_{e v}^{n-1} \pi_{*}^{s m} \mathbb{Q}[d] ;
$$

after tensoring with $\mathbb{C}$, it becomes isomorphic to the de Rham complex for $\mathcal{M}$, by the definition of mixed Hodge modules. Therefore,

$$
\operatorname{DR}_{P}(\mathcal{M}) \simeq j_{! *} \text { rat } V_{e v}^{n-1} \otimes_{\mathbb{Q}} \mathbb{C} .
$$


The purpose of this section is to prove that the hypercohomology $\mathbb{H}^{-d+1}\left(\mathrm{DR}_{P} \mathcal{M}\right)$ of the de Rham complex is isomorphic to the primitive cohomology of $X$.

Lemma 16.2. Assume that the vanishing cohomology of the hypersurfaces is nontrivial. Then the Leray spectral sequence gives rise to a (canonical) isomorphism

$$
H_{0}^{n}(X, \mathbb{C}) \simeq \mathbb{H}^{-d+1}\left(\operatorname{DR}_{P}(\mathcal{M})\right) .
$$

Proof. Since $\mathcal{R}=0$, we get $\mathcal{M}=\mathcal{M}_{e v}$. According to the results in 12 , we have

$$
H^{q} \pi_{*} \mathbb{Q}_{\mathscr{X}}^{H}\left[d_{\mathscr{X}}\right] \simeq \begin{cases}H^{n+q-1}(X) \otimes \mathbb{Q}_{P}^{H}[d] & \text { for } q<0, \\ H^{n+q+1}(X)(1) \otimes \mathbb{Q}_{P}^{H}[d] & \text { for } q>0, \\ j_{! *} V_{e v}^{n-1} \oplus H^{n-1}(X) \otimes \mathbb{Q}_{P}^{H}[d] & \text { for } q=0 .\end{cases}
$$

The $\mathcal{D}$-module component of $j_{! *} V_{e v}^{n-1}$ is precisely $\mathcal{M}$, and so the hypercohomology of $\operatorname{DR}_{P}(\mathcal{M})$ computes the complex vector spaces underlying the cohomology modules of $j_{! *} V_{e v}^{n-1}$. We calculate that

$$
\begin{aligned}
H^{-d+1} a_{P *} H^{0} \pi_{*} \mathbb{Q}_{\mathscr{X}}^{H}\left[d_{\mathscr{X}}\right] & \simeq H^{-d+1} a_{P *} j_{! *} V_{e v}^{n-1} \oplus H^{n-1}(X) \otimes H^{1} a_{P *} \mathbb{Q}_{P}^{H} \\
& =H^{-d+1} a_{P *} j_{! *} V_{e v}^{n-1},
\end{aligned}
$$

because $H^{1} a_{P *} \mathbb{Q}_{P}^{H}=H^{1}(P)=0$. It follows that $\mathbb{H}^{-d+1}\left(\operatorname{DR}_{P}(\mathcal{M})\right)$ is the complex vector space of the mixed Hodge structure $H^{-d+1} a_{P *} H^{0} \pi_{*} \mathbb{Q}_{\mathscr{X}}^{H}\left[d_{\mathscr{X}}\right]$.

Now we bring in the (perverse) Leray spectral sequence,

$$
E_{2}^{p, q}=H^{p} a_{P *} H^{q} \pi_{*} \mathbb{Q}_{\mathscr{X}}^{H}\left[d_{\mathscr{X}}\right] \Longrightarrow H^{p+q} a_{\mathscr{X} *} \mathbb{Q}_{\mathscr{X}}^{H}\left[d_{\mathscr{X}}\right]=H^{p+q+d_{\mathscr{X}}}(\mathscr{X}),
$$

which is a spectral sequence of mixed Hodge modules. Note that $E_{2}^{p, q}=0$ whenever $p<-d=-\operatorname{dim} P$. The term we are really interested in is

$$
E_{2}^{-d+1,0} \simeq H^{-d+1} a_{P *} j_{! *} V_{e v}^{n-1}
$$

it sits in degree $-d+1$, and is thus a graded quotient of $H^{-d+1+d \mathscr{X}}(\mathscr{X})=H^{n}(\mathscr{X})$.

The Decomposition Theorem implies that the spectral sequence degenerates at the $E_{2}$-page (in fact, it even implies that $H^{k} a_{\mathscr{X} *} \mathbb{Q}_{\mathscr{X}}^{H}\left[d_{\mathscr{X}}\right]$ is isomorphic to the direct sum of all the $E_{2}^{p, q}$ with $p+q=k$, albeit non-canonically). Let us write $L^{\bullet}$ for the induced filtration on the cohomology of $\mathscr{X}$. We then have a short exact sequence of mixed Hodge structures

$$
0 \longrightarrow L^{1} H^{n}(\mathscr{X}) \longrightarrow H^{n}(\mathscr{X}) \longrightarrow E_{2}^{-d, 1} \longrightarrow 0,
$$

and $E_{2}^{-d, 1} \simeq H^{n+2}(X)(1) \otimes H^{0}(P)$ by virtue of (16.3).

Consider now the pullback map $\phi^{*}: H^{n}(X) \rightarrow H^{n}(\mathscr{X})$. As the primitive cohomology is the kernel of $H^{n}(X) \rightarrow H^{n+2}(X)(1)$, we get an induced map from $H^{n}(X)_{\text {prm }}$ to $L^{1} H^{n}(\mathscr{X})$. The next step of the Leray filtration gives another short exact sequence

$$
0 \longrightarrow L^{2} H^{n}(\mathscr{X}) \longrightarrow L^{1} H^{n}(\mathscr{X}) \stackrel{\rho}{\longrightarrow} E_{2}^{-d+1,0} \longrightarrow 0
$$

and by composition, we finally obtain a (canonical) map of mixed Hodge structures

$$
\rho \circ \phi^{*}: H_{0}^{n}(X) \rightarrow E_{2}^{-d+1,0} \simeq H^{-d+1} a_{P *} j_{! *} V_{e v}^{n-1} .
$$

That this map is an isomorphism follows easily from the fact that $\phi: \mathscr{X} \rightarrow X$ is a projective space bundle of rank $d-1$. Indeed, we naturally have

$$
H^{n}(\mathscr{X}) \simeq \bigoplus_{i \geq 0} H^{i}(P) \otimes H^{n-i}(X)=H^{n}(X) \oplus \bigoplus_{i \geq 2} H^{i}(P) \otimes H^{n-i}(X) .
$$


The terms in the direct sum are precisely the graded quotients of $L^{2} H^{n}(\mathscr{X})$, because the isomorphisms in (16.3) imply that

$$
E_{2}^{-d+i,-i+1}=H^{-d+i} a_{P *} H^{-i-1} \pi_{*} \mathbb{Q}_{\mathscr{X}}^{H}\left[d_{\mathscr{X}}\right] \simeq H^{i}(P) \otimes H^{n-i}(X)
$$

whenever $i \geq 2$. Therefore, the map from $H^{n}(X)$ to $H^{n}(\mathscr{X}) / L^{2} H^{n}(\mathscr{X})$ is an isomorphism for dimension reasons; this implies that (16.4) is also an isomorphism. By passing to the underlying complex vector spaces, we get the result.

17. Cohomology sheaves of the de Rham complex. We conclude our application of the theory of mixed Hodge modules by determining the cohomology sheaves of the de Rham complex $\operatorname{DR}_{P}(\mathcal{M})$. Again, we assume throughout that the vanishing cohomology of the hypersurfaces is nontrivial, so that $\mathcal{M}_{e v}=\mathcal{M}$. Recall that $\operatorname{DR}_{P}(\mathcal{M})$ is a perverse complex, because $\mathcal{M}$ underlies a Hodge module; all of its cohomology sheaves

$$
\mathcal{H}^{k}=\mathcal{H}^{k} \operatorname{DR}_{P}\left(\mathcal{M}_{e v}\right) \simeq \mathcal{H}^{k} \operatorname{DR}_{P}(\mathcal{M})
$$

are therefore constructible sheaves (in the Zariski topology). The following lemma describes them very precisely.

Lemma 17.1. If the vanishing cohomology of the hypersurfaces is nontrivial, then

$$
R^{n-1+(d+k)} \pi_{*} \mathbb{C}_{\mathscr{X}} \simeq \mathcal{H}^{k} \operatorname{DR}_{P}(\mathcal{M}) \oplus H^{n-1-(d+k)}(X, \mathbb{C}) \otimes \mathbb{C}_{P}
$$

for all $k \geq-d$.

Proof. The proof mirrors that of Corollary 5.6 in [BFNP07. Let $p \in P$ be an arbitrary point. Since the map $\pi: \mathscr{X} \rightarrow P$ is proper, we have

$$
\left(R^{n-1+d+k} \pi_{*} \mathbb{C}_{\mathscr{X}}\right)_{p}=H^{n-1+d+k}\left(\mathscr{X}_{p}, \mathbb{C}\right)=\operatorname{rat} H^{n-1+d+k}\left(\mathscr{X}_{p}\right) \otimes_{\mathbb{Q}} \mathbb{C}
$$

for the stalk of the higher direct image sheaf at $p$, by the Proper Base Change Theorem from topology. Because of the decomposition in (10.1), we also have

$$
H^{n-1+d+k}\left(\mathscr{X}_{p}\right)=H^{d \mathscr{X}+k}\left(\mathscr{X}_{p}\right)=H_{p}^{k}\left(\pi_{*} \mathbb{Q}_{\mathscr{X}}^{H}\left[d_{\mathscr{X}}\right]\right)=\bigoplus_{i j} H_{p}^{k-i}\left(E_{i, j}\right) .
$$

The assumption on the vanishing cohomology implies that $E_{i, j}=0$ for all $j \neq 0$; see Proposition 15.1 and the work in \$12. Therefore

$$
H^{n-1+d+k}\left(\mathscr{X}_{p}\right)=\bigoplus_{i} H_{p}^{k-i}\left(E_{i, 0}\right) .
$$

The remaining terms are now easily computed. On the one hand,

$$
H_{p}^{k}\left(E_{0,0}\right) \simeq H_{p}^{k}\left(j_{! *} V^{n-1}\right) \simeq H_{p}^{k}\left(j_{! *} V_{e v}^{n-1}\right) \oplus \begin{cases}H^{n-1}(X) & \text { if } k=-d, \\ 0 & \text { otherwise }\end{cases}
$$

from Lemma 11.4. On the other hand, we have

$$
E_{i, 0}= \begin{cases}H^{n+i-1}(X) \otimes \mathbb{Q}_{P}^{H}[d] & \text { for } i<0, \\ H^{n-i-1}(X)(-i) \otimes \mathbb{Q}_{P}^{H}[d] & \text { for } i>0,\end{cases}
$$

again using the results in $₫ 12$. Since $H_{p}^{k-i}\left(\mathbb{Q}_{P}^{H}[d]\right)=0$ for $k-i \neq-d$, it then follows that

$$
H_{p}^{k-i}\left(E_{i, 0}\right) \simeq \begin{cases}H^{n-1+(d+k)}(X) & \text { if } i=d+k<0 \\ H^{n-1-(d+k)}(X)(-(d+k)) & \text { if } i=d+k>0 \\ 0 & \text { if } i \neq d+k \text { and } i \neq 0\end{cases}
$$


In conclusion, we have for $k \geq-d$ an isomorphism

$$
H^{n-1+(d+k)}\left(\mathscr{X}_{p}\right) \simeq H_{p}^{k}\left(j_{! *} V_{e v}^{n-1}\right) \oplus H^{n-1-(d+k)}(X)(-(d+k)) .
$$

Now apply the functor rat to this, and note that

$$
\operatorname{rat} H_{p}^{k}\left(j_{! *} V_{e v}^{n-1}\right) \otimes_{\mathbb{Q}} \mathbb{C} \simeq \mathcal{H}_{p}^{k}
$$

by the comments preceding the statement of the lemma. On stalks, we thus have

$$
\left(R^{n-1+(d+k)} \pi_{*} \mathbb{C}_{\mathscr{X}}\right)_{p} \simeq \mathcal{H}_{p}^{k} \oplus H^{n-1-(d+k)}(X, \mathbb{C}),
$$

from which the asserted identity follows immediately.

Note. It should be noted that the second part of the isomorphism in (17.2) can be described explicitly. Let $L: H^{k}(X) \rightarrow H^{k+2}(X)(1)$ be the Lefschetz operator associated with the very ample line bundle $\mathscr{O}_{X}(1)$. For each hypersurface $\mathscr{X}_{p} \subseteq X$, smooth or not, there is then naturally a map

$$
H^{n-1-(d+k)}(X)(-(d+k)) \stackrel{L^{d+k}}{\longrightarrow} H^{n-1+(d+k)}(X) \longrightarrow H^{n-1+(d+k)}\left(\mathscr{X}_{p}\right) .
$$

So (17.2) tells us in particular that this map is always injective.

\section{Properties of the Hodge filtration}

Note. For the remainder of the paper, we assume that the vanishing cohomology of the hypersurfaces is nontrivial; this guarantees that $\mathcal{M}_{e v}=\mathcal{M}$.

18. The lowest level in the filtration. Our next result is about the lowest level in the filtration on $\mathcal{M}$, namely the sheaf $F_{1} \mathcal{M}$, and its relationship to the relative canonical bundle of $\mathscr{X} \rightarrow P$. In this context, the following theorem by Kawamata comes to mind: If $f: Y \rightarrow X$ is an algebraic fiber space such that $f$ is smooth over the complement of a normal crossing divisor $D$, and $R^{\operatorname{dim} Y-\operatorname{dim} X} f_{*} \mathbb{C}$ has unipotent monodromy on $X \backslash D$, then $f_{*} \mathscr{O}_{Y}\left(K_{Y / X}\right)$ is locally free and nef, and agrees with the lowest level of the Hodge filtration on the canonical extension [Kaw81, p. 266]. Obviously, neither of the two assumptions is satisfied in our case, but a similar result holds.

Proposition 18.1. Assume that $\mathscr{O}_{X}(1)$ is sufficiently ample. Then $F_{1} \mathcal{M}$ is an ample vector bundle, and forms part of a short exact sequence

$$
0 \longrightarrow F_{1} \mathcal{M} \longrightarrow \pi_{*} \mathscr{O}_{\mathscr{X}}\left(K_{\mathscr{X} / P}\right) \rightarrow H^{n-1,0}(X) \otimes \mathscr{O}_{P} \longrightarrow 0 .
$$

In particular, $\pi_{*} \mathscr{O}_{\mathscr{X}}\left(K_{\mathscr{X} / P}\right)$ is locally free and nef.

Proof. From the locally free resolution introduced in $\$ 14$, we get a short exact sequence

$$
0 \longrightarrow H^{0}\left(X, \Omega_{X}^{n}\right) \otimes \mathscr{O}_{P} \longrightarrow H^{0}\left(X, \Omega_{X}^{n}(1)\right) \otimes \mathscr{O}_{P}(1) \longrightarrow F_{1} \mathcal{M} \longrightarrow 0 .
$$

The first map is pointwise injective, because $H^{0}\left(X, \Omega_{X}^{n}\right) \subseteq H^{0}\left(X, \Omega_{X}^{n}\left(\mathscr{X}_{p}\right)\right)$ for every $p \in P$, and so the quotient $F_{1} \mathcal{M}$ is locally free, proving the first assertion.

To establish (18.2), we use the fact that $\mathscr{X}$ is a smooth hypersurface in the product $P \times X$, with line bundle $\mathscr{O}_{P \times X}(\mathscr{X})=\mathscr{O}_{P \times X}(1)$. Thus the relative canonical bundle for $\pi: \mathscr{X} \rightarrow P$ is given by the formula

$$
\mathscr{O}_{\mathscr{X}}\left(K_{\mathscr{X} / P}\right) \simeq \phi^{*} \Omega_{X}^{n} \otimes \mathscr{O}_{\mathscr{X}}(1),
$$

where $\phi: \mathscr{X} \rightarrow X$ is the second projection. Pushing forward the exact sequence

$$
0 \longrightarrow p r_{X}^{*} \Omega_{X}^{n} \longrightarrow p r_{X}^{*} \Omega_{X}^{n} \otimes \mathscr{O}_{P \times X}(1) \longrightarrow \phi^{*} \Omega_{X}^{n} \otimes \mathscr{O}_{\mathscr{X}}(1) \longrightarrow 0
$$


and using the vanishing of $H^{1}\left(X, \Omega_{X}^{n}(1)\right)$, we get a five-term exact sequence; the second assertion follows by comparing it with the resolution for $F_{1} \mathcal{M}$ in (18.3).

Now $F_{1} \mathcal{M}$ is evidently ample, since it is a quotient of $H^{0}\left(X, \Omega_{X}^{n}(1)\right) \otimes \mathscr{O}_{P}(1)$. Because of the short exact sequence in (18.2), it is then immediate that the direct image $\pi_{*} \mathscr{O}_{\mathscr{X}}\left(K_{\mathscr{X} / P}\right)$ is both locally free and nef.

Note. A similar result is true in certain other cases: in the 34-dimensional family of all cubic threefolds in $\mathbb{P}^{4}$, for instance, the coherent sheaf $F_{2} \mathcal{M}$ is locally free, and in fact isomorphic to $H^{0}\left(\mathbb{P}^{4}, \mathscr{O}_{\mathbb{P}^{4}}(1)\right) \otimes \mathscr{O}_{P}(2)$.

19. Computation of Ext-groups. The purpose of this section is the computation of the groups $\operatorname{Ext}_{P}^{i}\left(F_{k} \mathcal{M}, \mathscr{O}_{P}\right)$. We begin by looking at the sheaves $F_{k-n-1} \mathcal{N}^{0}$.

Lemma 19.1. We have

$$
\operatorname{Ext}_{P}^{i}\left(F_{k-n-1} \mathcal{N}^{0}, \mathscr{O}_{P}\right) \simeq \begin{cases}0 & \text { for } i=0,1, \\ \left(\frac{F^{n+1-k} H^{n+1-i}(X, \mathbb{C})}{F^{n-k} H^{n-1-i}(X, \mathbb{C})}\right)^{\vee} & \text { for } 2 \leq i \leq d-1 .\end{cases}
$$

Proof. We use the locally free complex $\mathscr{E}_{k} \bullet$ from $\$ 14$, its cohomology sheaf in degree $i$ is the sheaf $F_{k-n-1} \mathcal{N}^{i}$. When we apply the functor $\operatorname{Hom}_{P}\left(-, \mathscr{O}_{P}\right)$ to the complex, we obtain two spectral sequences; the terms of the first one are

$$
{ }^{\prime} E_{1}^{p, q}=\operatorname{Ext}_{P}^{q}\left(\mathscr{E}_{k}^{-p}, \mathscr{O}_{P}\right) \simeq H^{d-q}\left(P, \mathscr{E}_{k}^{-p} \otimes \mathscr{O}_{P}(-d-1)\right)^{\vee},
$$

by Serre Duality. Now each $\mathscr{E}_{k}^{-p}$ is either zero (when $k-p \leq 0$ ), or a sum of positive line bundles $\mathscr{O}_{P}(k-p)$, and so ' $E_{1}^{p, q}$ can only be nonzero if $q=d$ and $0 \leq p \leq k-1-d$. The limit of the spectral sequence is therefore zero whenever $p+q<d$. We conclude that the second spectral sequence, with terms

$$
{ }^{\prime \prime} E_{2}^{p, q}=\operatorname{Ext}_{P}^{p}\left(F_{k-n-1} \mathcal{N}^{-q}, \mathscr{O}_{P}\right)
$$

converges to zero in degrees $p+q<d$. For $q<0$, each $F_{k-n-1} \mathcal{N}^{-q}$ is a trivial bundle, and so ${ }^{\prime \prime} E_{2}^{p, q}=0$ unless $p=0$ or $q=0$. This implies that ${ }^{\prime \prime} E_{2}^{0, p-1} \simeq{ }^{\prime \prime} E_{2}^{p, 0}$ for $2 \leq p \leq d-1$. Since ${ }^{\prime \prime} E_{2}^{p, 0}=\operatorname{Ext}_{P}^{p}\left(F_{k-n-1} \mathcal{N}^{0}, \mathscr{O}_{P}\right)$ and

$$
{ }^{\prime \prime} E_{2}^{0, p-1}=\operatorname{Hom}\left(F_{k-n-1} \mathcal{N}^{1-p}, \mathscr{O}_{P}\right) \simeq\left(\frac{F^{n+1-k} H^{n+1-p}(X, \mathbb{C})}{F^{n-k} H^{n-1-p}(X, \mathbb{C})}\right)^{\vee}
$$

by (14.1), we get the assertion.

Theorem 19.2. For all integers $0 \leq i \leq d-1$, we have

$$
\operatorname{Ext}_{p}^{i}\left(F_{k} \mathcal{M}, \mathscr{O}_{P}\right) \simeq\left(\frac{F^{n+1-k} H^{n+1-i}(X, \mathbb{C})}{F^{n-k} H^{n-1-i}(X, \mathbb{C})}\right)^{\vee}
$$

In particular, $\operatorname{Hom}_{P}\left(F_{k} \mathcal{M}, \mathscr{O}_{P}\right)=0$, and $\operatorname{Ext}_{P}^{1}\left(F_{k} \mathcal{M}, \mathscr{O}_{P}\right) \simeq\left(F^{n+1-k} H_{0}^{n}(X, \mathbb{C})\right)^{\vee}$.

Proof. The short exact sequence in Corollary 13.10 becomes

$$
0 \longrightarrow F^{n+1-k} H_{0}^{n}(X, \mathbb{C}) \otimes \mathscr{O}_{P} \longrightarrow F_{k-n-1} \mathcal{N}^{0} \longrightarrow F_{k} \mathcal{M} \longrightarrow 0
$$

after passing to $F_{k-n-1}$. The result now follows from Lemma 19.1 by looking at the long exact sequence for the functor $\operatorname{Hom}_{P}\left(-, \mathscr{O}_{P}\right)$. 
The proof shows that the isomorphism between $\operatorname{Ext}_{P}^{1}\left(F_{k} \mathcal{M}, \mathscr{O}_{P}\right)$ and the group $\left(F^{n+1-k} H_{0}^{n}(X, \mathbb{C})\right)^{\vee}$ is given by the extension class of the sequence (19.3). This class is an element $\varepsilon_{k} \in \operatorname{Ext}_{P}^{1}\left(F_{k} \mathcal{M}, F^{n+1-k} H_{0}^{n}(X, \mathbb{C}) \otimes \mathscr{O}_{P}\right)$. Any linear map $f: F^{n+1-k} H_{0}^{n}(X, \mathbb{C}) \rightarrow \mathbb{C}$ defines a homomorphism

$$
f_{*}: \operatorname{Ext}_{P}^{1}\left(F_{k} \mathcal{M}, F^{n+1-k} H_{0}^{n}(X, \mathbb{C}) \otimes \mathscr{O}_{P}\right) \rightarrow \operatorname{Ext}_{P}^{1}\left(F_{k} \mathcal{M}, \mathscr{O}_{P}\right),
$$

and $f_{*}\left(\varepsilon_{k}\right)$ is the element corresponding to $f$ under the isomorphism in the theorem.

Note. Likewise, we have $\operatorname{Ext}_{P}^{1}\left(\operatorname{Gr}_{k}^{F} \mathcal{M}, \mathscr{O}_{P}\right) \simeq H_{0}^{n+1-k, k-1}(X)$.

20. Duality theorems. In this section, we apply the general duality theorem of Sch09a to the filtered $\mathcal{D}$-module $(\mathcal{M}, F)$. Note that $\left(\mathcal{M}_{e v}, F\right)$ underlies a polarized Hodge module of weight $\operatorname{dim} \mathscr{X}=d+n-1$ on $P$, and that we have $F_{k} \mathcal{M}_{e v}=$ $F_{k+n} \mathcal{M}$. Since we have already computed the characteristic module

$$
\mathscr{C}(\mathcal{M}, F) \simeq \psi^{*} \Omega_{X}^{n}
$$

in Corollary 15.7 the following result is a direct consequence of the duality theorem. As for notation, we let $\psi: \mathscr{Y} \rightarrow X$ and $\pi: \mathscr{Y} \rightarrow P$ denote the two projection maps, and set $\mathscr{C}=\mathscr{C}(\mathcal{M}, F)$ and $\mathscr{G}_{k}=\mathrm{Gr}_{k}^{F} \mathcal{M}$ for $k \in \mathbb{Z}$.

Theorem 20.1. For every $k \in \mathbb{Z}$, we have an exact sequence

$$
\mathscr{H} O{ }^{\prime}\left(\mathscr{G}_{n+1-k}, \mathscr{O}_{P}\right) \hookrightarrow \mathscr{G}_{k} \longrightarrow \pi_{*}\left(\mathscr{C} \otimes \mathscr{O}_{\mathscr{Y}}(k)\right) \longrightarrow \mathscr{E} x t^{1}\left(\mathscr{G}_{n+1-k}, \mathscr{O}_{P}\right),
$$

as well as isomorphisms

$$
R^{i} \pi_{*}\left(\mathscr{C} \otimes \mathscr{O}_{\mathscr{Y}}(k)\right) \simeq \mathscr{E} x t^{i+1}\left(\mathscr{G}_{n+1-k}, \mathscr{O}_{P}\right)
$$

for every $i \geq 1$.

The proof in Sch09a is based on the fact that $\operatorname{Gr}^{F} \mathcal{M}$ is Cohen-Macaulay as a graded module over the symmetric algebra $\operatorname{Sym} \Theta_{P}$. It is also possible to derive the theorem from a careful analysis of the spectral sequence

$$
E_{1}^{p, q}=R^{q} \pi_{*}\left(\phi^{*} \Omega_{X}^{p}(k-n+p)\right) \otimes \mathscr{O}_{P}(k-n+p) \Longrightarrow R^{p+q-n} \pi_{*}\left(\psi^{*} \Omega_{X}^{n} \otimes \mathscr{O}_{\mathscr{Y}}(k)\right),
$$

coming from the resolution in Lemma 15.2, details can be found in Sch08. We note that the first map in (20.2) is induced by the intersection pairing: its restriction to $P^{s m}$ is a map of vector bundles

$$
\left(F^{k-1} \mathscr{H}_{\mathscr{O}} / F^{k} \mathscr{H}_{\mathscr{O}}\right)^{\vee} \rightarrow F^{n-k} \mathscr{H}_{\mathscr{O}} / F^{n-k+1} \mathscr{H}_{\mathscr{O}},
$$

which, fiber by fiber, is given by $(2 \pi i)^{n-1}$ times integration over the smooth hypersurface $\mathscr{X}_{p}$ (up to a sign factor).

Since the duality theorem actually produces an exact triangle in the derived category $D^{b}(P)$, we can take the push-forward to a point to arrive at the following global statement. Note that $\operatorname{Hom}_{P}\left(\operatorname{Gr}_{n+1-k}^{F} \mathcal{M}\right)=0$ by Theorem 19.2

Proposition 20.4. For each $k \in \mathbb{Z}$, we have an exact sequence

$$
H^{0}\left(P, \operatorname{Gr}_{k}^{F} \mathcal{M}\right) \hookrightarrow H^{0}\left(\mathscr{Y}, \psi^{*} \Omega_{X}^{n} \otimes \mathscr{O}_{\mathscr{Y}}(k)\right) \longrightarrow \operatorname{Ext}_{P}^{1}\left(\operatorname{Gr}_{n+1-k}^{F} \mathcal{M}, \mathscr{O}_{P}\right) .
$$

Moreover, we have $\operatorname{Ext}_{P}^{i+1}\left(\operatorname{Gr}_{n+1-k}^{F} \mathcal{M}, \mathscr{O}_{P}\right) \simeq H^{i}\left(\mathscr{Y}, \psi^{*} \Omega_{X}^{n} \otimes \mathscr{O}_{\mathscr{Y}}(k)\right)$ for all $i \geq 1$ and all values of $k$. 
21. A curious vanishing theorem. We digress to point out a curious application of the computations for the groups $\operatorname{Ext}_{P}^{i}\left(\operatorname{Gr}_{k}^{F} \mathcal{M}, \mathscr{O}_{P}\right)$ when $i>0$. We have obtained two different expressions for these groups, one in Proposition 20.4, the other in Theorem 19.2. By comparing the two, we get the following statement (which, I believe, is originally due to M. Green).

Proposition 21.1. Let $X$ be a smooth projective variety of dimension $n$, and let $\mathscr{O}_{X}(1)$ be a sufficiently ample line bundle on $X$. For all $k \geq 0$ and $q>0$, we have

$$
H^{q}\left(X, \Omega_{X}^{n} \otimes \operatorname{Sym}^{k} N_{X \mid Q}\right) \simeq H^{q}\left(\mathscr{Y}, \psi^{*} \Omega_{X}^{n} \otimes \mathscr{O}_{\mathscr{Y}}(k)\right) \simeq H_{0}^{n-k, q+k}(X),
$$

where $N_{X \mid Q}$ is the normal bundle for the embedding of $X$ into projective space.

Proof. Recall that $\psi: \mathscr{Y} \rightarrow X$ is the projective bundle $\mathbb{P}\left(N_{X \mid Q}\right)$. For $k \geq 0$, we thus have $\psi_{*} \mathscr{O}_{\mathscr{Y}}(k) \simeq \operatorname{Sym}^{k} N_{X \mid Q}$. The first asserted isomorphism is then obtained by push-forward along the map $\psi$. The second isomorphism now follows immediately by combining the result of Proposition 20.4 and Theorem 19.2

In particular, one gets a vanishing theorem for the ample vector bundle $N_{X \mid Q}$. For example, the condition $q+k \geq n+1$ is sufficient to make the cohomology group $H^{q}\left(X, \Omega_{X}^{n} \otimes \operatorname{Sym}^{k} N_{X \mid Q}\right)$ be zero. This does not seem to follow from the standard vanishing theorems for ample vector bundles, such as Griffiths' Theorem [Laz04b, Theorem 7.3.1 on p. 90], because the factor of $\operatorname{det} N_{X \mid Q}$ is missing. At the same time, whenever $X$ has nontrivial primitive cohomology in degree $(n-k, q+k)$, one gets an example where the group in question is not zero. This places restrictions on the kind of vanishing theorem one can get for general ample vector bundles.

22. The Serre conditions. The sheaves $F_{k} \mathcal{M}$ are natural extensions of the Hodge bundles $F^{n-k} \mathscr{H}_{\mathscr{O}}$ on $P^{s m}$. While they cannot in general be locally free, we nevertheless expect that $F_{k} \mathcal{M}$ should have good properties when the line bundle $\mathscr{O}_{X}(1)$ is sufficiently ample - basically because the complexity of the dual variety appears in very high codimension. In this section, we illustrate this by proving that each $F_{k} \mathcal{M}$ in the range $1 \leq k \leq n$ is a reflexive sheaf.

More precisely, recall that a coherent sheaf $\mathscr{F}$ on a nonsingular algebraic variety $P$ is said to satisfy (a slight modification of) Serre's condition $S_{m}$, if the inequality

$$
\operatorname{depth} \mathscr{F}_{p} \geq \min \left(m, \operatorname{dim} \mathscr{O}_{P, p}\right)
$$

holds at every point $p \in P$. A locally free sheaf satisfies $S_{m}$ for all values of $m$; on the other hand, condition $S_{1}$ is equivalent to being torsion-free, and $S_{2}$ to being reflexive. There is also a useful criterion for verifying Serre's condition: $\mathscr{F}$ satisfies $S_{m}$ if and only if, for every $i>0$, the codimension of the support of $\mathscr{E} x t^{i}\left(\mathscr{F}, \mathscr{O}_{P}\right)$ is at least $i+m$ (see Pop09, Proposition 7.5] for a careful proof). In particular, $\mathscr{F}$ is then locally free in codimension $m$.

The following theorem shows that the sheaves $\operatorname{Gr}_{k}^{F} \mathcal{M}$ and $F_{k} \mathcal{M}$ are well-behaved when $1 \leq k \leq n$, at least when $\mathscr{O}_{X}(1)$ is sufficiently ample.

Theorem 22.1. Fix an integer $m \geq 1$. If the line bundle $\mathscr{O}_{X}(1)$ is ample enough, then each sheaf $\mathrm{Gr}_{k}^{F} \mathcal{M}$ in the interval $1 \leq k \leq n$ satisfies Serre's condition $S_{m}$.

The proof is based on bounding the codimension of the set of hyperplane sections with many singular points, or with singular points of high multiplicity.

Proposition 22.2. Fix two positive integers $N$ and $r$. If $\mathscr{O}_{X}(1)$ is sufficiently ample, then the linear system $P$ has the following properties: 
(a) The subset $P_{1}(N) \subseteq P$ of hypersurfaces with at least $N$ singular points has codimension at least $N$ in $P$.

(b) The subset $P_{2}(r) \subseteq P$ of hypersurfaces with a singular point of multiplicity at least $r$ has codimension at least $r-1$ in $P$.

Proof. As usual, let $d=\operatorname{dim} P$. To prove(a) let $\mathfrak{S}_{1}(N) \subseteq P \times X^{N}$ be the closure of the set of points $\left(p, x_{1}, \ldots, x_{N}\right)$ for which $x_{1}, \ldots, x_{N}$ are distinct singular points of the hypersurface $\mathscr{X}_{p}$. Then $\mathfrak{S}_{1}(N)$ is irreducible, and its fiber over a general point $\left(x_{1}, \ldots, x_{N}\right) \in X^{N}$ has dimension $d-N(n+1)$ by Lemma 22.3. provided that $\mathscr{O}_{X}(1)$ is sufficiently ample. Therefore $\operatorname{dim} \mathfrak{S}_{1}(N)=d-N$, and so its projection to $P$, which equals $P_{1}(N)$, has dimension at most $d-N$.

The argument for (b) is similar; this time, consider the set $\mathfrak{S}_{2}(r) \subseteq P \times X$ of points $(p, x)$ such that $x \in \mathscr{X}_{p}$ is a singular point of multiplicity at least $r$. By Lemma 22.3, we can take $\mathscr{O}_{X}(1)$ sufficiently ample to separate $r$-jets; then the fiber of $\mathfrak{S}_{2}(r)$ over a point $x \in X$ has dimension at most $d-\left(\begin{array}{c}n+r-1 \\ r-1\end{array}\right)$, and so the dimension of $P_{2}(r)$ cannot be greater than

$$
n+d-\left(\begin{array}{c}
n+r-1 \\
r-1
\end{array}\right) \leq d-r+1
$$

During the proof, we used the following lemma about separation of jets; it is a straightforward generalization of [Laz04a, Theorem 5.1.17].

Lemma 22.3. Fix two positive integers $N$ and $r$. If $\mathscr{O}_{X}(1)$ is sufficiently ample, then for any collection of $N$ distinct points $x_{1}, \ldots, x_{N} \in X$, the restriction map

$$
H^{0}\left(X, \mathscr{O}_{X}(1)\right) \rightarrow \bigoplus_{i=1}^{N} \mathscr{O}_{X}(1) \otimes \mathscr{O}_{X, x_{i}} / \mathfrak{m}_{x_{i}}^{r+1}
$$

is surjective.

We now turn to the proof of Theorem 22.1.

Proof. It suffices to show that the sheaves $\mathscr{E} x t^{i}\left(\operatorname{Gr}_{n+1-k}^{F} \mathcal{M}, \mathscr{O}_{P}\right)$ are supported in codimension at least $i+m$, for all $i>0$. First, we treat the case when $i \geq 2$, where we have

$$
\mathscr{E} x t^{i}\left(\operatorname{Gr}_{n+1-k}^{F} \mathcal{M}, \mathscr{O}_{P}\right) \simeq R^{i-1} \pi_{*}\left(\psi^{*} \Omega_{X}^{n} \otimes \mathscr{O} \mathscr{Y}(k)\right)
$$

by (20.3). The support of this sheaf is therefore contained in the locus $P_{1}$ where the singular set of the fibers has positive dimension; by Proposition 22.2, we can make its codimension greater than $i+m$ by taking $\mathscr{O}_{X}(1)$ sufficiently ample.

What remains is the case $i=1$. The exact sequence in (20.2) shows that $\mathscr{E} x t^{1}\left(\mathrm{Gr}_{n+1-k} \mathcal{M}, \mathscr{O}_{P}\right)$ is the cokernel of the restriction map

$$
\operatorname{Gr}_{k}^{F} \mathcal{M} \rightarrow \pi_{*}\left(\psi^{*} \Omega_{X}^{n} \otimes \mathscr{O} \mathscr{Y}(k)\right) .
$$

To complete the proof, we have to show that this map is surjective except on a set of codimension at least $m+1$. We may restrict our attention to the open subset $P \backslash P_{1}$ where $\pi: \mathscr{Y} \rightarrow P$ has finite fibers; over that set, $\pi$ is a finite morphism, and so the stalk of $\pi_{*}\left(\psi^{*} \Omega_{X}^{n} \otimes \mathscr{O}_{\mathscr{Y}}(k)\right)$ at a point $p \in P \backslash P_{1}$ equals $H^{0}\left(\mathscr{Y}_{p}, \Omega_{X}^{n}(k)\right)$. Since we know from Corollary 14.4 that $H^{0}\left(X, \Omega_{X}^{n}(k)\right) \otimes \mathscr{O}_{P}(k) \rightarrow \mathrm{Gr}_{k}^{F} \mathcal{M}$ is always surjective, it is therefore sufficient to prove that the map

$$
H^{0}\left(X, \Omega_{X}^{n}(k)\right) \rightarrow H^{0}\left(\mathscr{Y}_{p}, \Omega_{X}^{n}(k)\right)
$$

is surjective, except when $p \in P \backslash P_{1}$ lies in a subset of codimension $\geq m+1$. 
Let $P_{2} \subseteq P$ be the set of hyperplane sections $\mathscr{X}_{p}$ that either have more than $m$ singular points, or have a singular point of multiplicity greater than $m+1$; by Proposition 22.2. the codimension of $P_{2}$ is at least $m+1$. On the other hand, (22.5) is surjective for each $p \in P \backslash P_{2}$; indeed, $\mathscr{Y}_{p}$ consists of at most $m$ points, each with multiplicity no greater than $m+1$, and so we can apply Lemma 22.3 This concludes the proof that $\operatorname{Gr}_{k}^{F} \mathcal{M}$ satisfies Serre's condition $S_{m}$.

Corollary 22.6. If $\mathscr{O}_{X}(1)$ is sufficiently ample, then for each $k=1, \ldots, n$, the sheaves $\operatorname{Gr}_{k}^{F} \mathcal{M}$ and $F_{k} \mathcal{M}$ are reflexive.

\section{REFERENCES}

[BBD82] A. A. Beǐlinson, J. Bernstein, and P. Deligne, Faisceaux pervers, Analysis and topology on singular spaces, I (Luminy, 1981), Astérisque, vol. 100, Société Mathématique de France, Paris, 1982, pp. 5-171.

$\left[\mathrm{BGK}^{+} 87\right]$ A. Borel, P.-P. Grivel, B. Kaup, A. Haefliger, B. Malgrange, and F. Ehlers, Algebraic D-modules, Perspectives in Mathematics, vol. 2, Academic Press Inc., Boston, MA, 1987.

[BFNP07] P. Brosnan, H. Fang, Z. Nie, and G. Pearlstein, Singularities of admissible normal functions (2007), available at arXiv:0711.0964. To appear in Inventiones Mathematicae.

[dCM07] M. A. A. de Cataldo and L. Migliorini, A remark on singularities of primitive cohomology classes (2007), available at arXiv:0711.1307v1

[DS09] A. Dimca and M. Saito, Vanishing cycle sheaves of one-parameter smoothings and quasi-semistable degenerations (2009), available at arXiv:0810.4896v2

[Gre85] M. L. Green, The period map for hypersurface sections of high degree of an arbitrary variety, Compositio Mathematica 55 (1985), no. 2, 135-156.

[GG07] M. L. Green and P. Griffiths, Algebraic cycles and singularities of normal functions, Algebraic cycles and motives (Grenoble, 2007), London Mathematical Society Lecture Note Series, vol. 343, Cambridge University Press, 2007, pp. 206-263.

[GG06] _ Algebraic cycles and singularities of normal functions. II, Inspired by S. S. Chern, Nankai Tracts in Mathematics, vol. 11, 2006, pp. 179-268.

[GMV94] M. L. Green, J. Murre, and C. Voisin, Algebraic cycles and Hodge theory, Lecture Notes in Mathematics, vol. 1594, Springer-Verlag, Berlin, 1994.

[Gri69] P. A. Griffiths, On the periods of certain rational integrals. I, II, Annals of Mathematics 90 (1969), 460-495 and 496-541.

[GS75] P. Griffiths and W. Schmid, Recent developments in Hodge theory: a discussion of techniques and results, Discrete subgroups of Lie groups and applications to moduli, Oxford University Press, Bombay, 1975, pp. 31-127.

[Kaw81] Y. Kawamata, Characterization of abelian varieties, Compositio Mathematica 43 (1981), no. 2, 253-276.

[Laz04a] R. Lazarsfeld, Positivity in algebraic geometry. I, Ergebnisse der Mathematik und ihrer Grenzgebiete. 3. Folge. A Series of Modern Surveys in Mathematics, vol. 48, Springer-Verlag, Berlin, 2004. Classical setting: line bundles and linear series.

[Laz04b] Positivity in algebraic geometry. II, Ergebnisse der Mathematik und ihrer Grenzgebiete. 3. Folge. A Series of Modern Surveys in Mathematics, vol. 49, SpringerVerlag, Berlin, 2004. Positivity for vector bundles, and multiplier ideals.

[Lau85] G. Laumon, Transformations canoniques et spécialisation pour les $\mathcal{D}$-modules filtrés, Differential systems and singularities (Luminy, 1983), 1985, pp. 56-129.

[Pop09] M. Popa, Generic vanishing filtrations and perverse objects in derived categories of coherent sheaves (2009), available at arXiv:0911.3648.

[Sai88] M. Saito, Modules de Hodge polarisables, Publications of the Research Institute for Mathematical Sciences 24 (1988), no. 6, 849-995.

[Sai90] Mixed Hodge modules, Publications of the Research Institute for Mathematical Sciences 26 (1990), no. 2, 221-333.

[Sai93] $\quad$ On b-function, spectrum and rational singularity, Mathematische Annalen 295 (1993), no. 1, 51-74. 
[Sch08] C. Schnell, The boundary behavior of cohomology classes and singularities of normal functions, Ph. D. thesis, Ohio State University, 2008. Available at OhioLink ETD

[Sch09a] , Local duality and polarized Hodge modules (2009), submitted, available at arxiv:0904.3480

[Sch09b] Complex analytic Néron models for arbitrary families of intermediate Jacobians (2009), submitted, available at arXiv:0910.0662v2

[Voi02] C. Voisin, Théorie de Hodge et géométrie algébrique complexe, Cours Spécialisés, vol. 10, Société Mathématique de France, Paris, 2002.

Department of Mathematics, Statistics \& Computer Science, University of Illinois at Chicago, 851 South Morgan Street, Chicago, IL 60607

E-mail address: cschnell@math.uic.edu 